\title{
Conservative dynamics of two-body systems at the fourth post-Newtonian approximation of general relativity
}

\author{
Thibault Damoun \\ Institut des Hautes Etudes Scientifiques, 35 route de Chartres, 91440 Bures-sur-Yvette, France \\ Piotr Jaranowsk $\oplus$ \\ Faculty of Physics, University of Biatystok, Ciotkowskiego 1L, 15-245 Biatystok, Poland \\ Gerhard Schäfer用 \\ Theoretisch-Physikalisches Institut, Friedrich-Schiller-Universität Jena, Max-Wien-Pl. 1, 07743 Jena, Germany
}

(Dated: 8 March, 2016)

\begin{abstract}
The fourth post-Newtonian (4PN) two-body dynamics has been recently tackled by several different approaches: effective field theory, Arnowitt-Deser-Misner Hamiltonian, action-angle-Delaunay averaging, effective-one-body, gravitational self-force, first law of dynamics, and Fokker action. We review the achievements of these approaches and discuss the complementarity of their results. Our main conclusions are: (i) the results of the first complete derivation of the 4PN dynamics [T. Damour, P. Jaranowski, and G. Schäfer, Phys. Rev. D 89, 064058 (2014)] have been, piecewise, fully confirmed by several subsequent works; (ii) the results of the Delaunay-averaging technique [T. Damour, P. Jaranowski, and G. Schäfer, Phys. Rev. D 91, 084024 (2015)] have been confirmed by several independent works; and (iii) several claims in a recent harmonic-coordinates Fokker-action computation [L. Bernard et al., arXiv:1512.02876v2 [gr-qc]] are incorrect, but can be corrected by the addition of a couple of ambiguity parameters linked to subtleties in the regularization of infrared and ultraviolet divergences.
\end{abstract}

\section{INTRODUCTION}

The general-relativistic two-body problem has acquired a renewed importance in view of the impending prospect of detecting the gravitational wave signals emitted by inspiraling and coalescing compact binaries. After the successful completion, 15 years ago, of the derivation of the third post-Newtonian (3PN) two-body dynamics [1] (see Refs. [2 5] for later rederivations), a natural challenge was to tackle the (conservative) fourth postNewtonian $(4 \mathrm{PN})$ dynamics. Several partial steps in the derivation of the $4 \mathrm{PN}$ dynamics have been performed [614], and culminated in the recent first full derivation, within the Arnowitt-Deser-Misner (ADM) Hamiltonian formulation of general relativity, of the 4PN dynamics in Ref. [16], completed by an action-angle-Delaunay averaging study in Ref. [17]. A remarkable feature of the conservative 4PN dynamics of Ref. [16] is the presence of a time-symmetric nonlocal-in-time interaction, which is directly related to the $4 \mathrm{PN}$-level tail-transported (retarded) interaction first discussed in Ref. [18] (see also Refs. [19, 20] for recent rediscussions).

In a recent preprint [21] Bernard et al. reported the computation of the Fokker action describing the 4PN dynamics in harmonic coordinates. They made several claims in (version 2 of) their preprint, notably (i) the

\footnotetext{
* damour@ihes.fr

†.jaranowski@uwb.edu.pl

$\ddagger$ gos@tpi.uni-jena.de

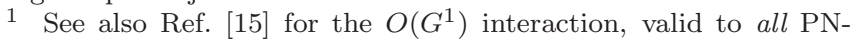
orders.
}

presence of a unique ambiguity, of infrared (IR) origin, parametrized by the parameter $\alpha$, and (ii) the need to choose the value $\alpha=\alpha_{\mathrm{B}^{3} \mathrm{FM}}=811 / 672$ to reproduce the analytically known [13] 4PN interaction energy for circular orbits. Moreover, they stated that their 4PN dynamics disagrees with the one recently derived by us [14, 16, 17] and that part of the discrepancy comes from our treatment of the nonlocal contribution to the dynamics.

The first aims of the present paper will be: 1) to show that the claims, denoted as (i) and (ii) above, of Ref. 21] are incorrect; 2) to suggest that the discrepancies between their result and ours is due both to their incorrect evaluation of the conserved energy of the nonlocal $4 \mathrm{PN}$ dynamics and to the need to complete their result by at least one further contribution parametrized by an additional ambiguity parameter, denoted $a$ below. We, however, emphasize that our suggested corrections represent only two rather minor adjustments among hundreds of terms that agree between two very difficult (four-loop level!) independent calculations, using different methods and different gauges. The other aims of our paper will be: 3) to explain in more detail than in our original paper [17] the logical basis and consistency of our reduction of the nonlocal 4PN dynamics to a formally local action-angle Hamiltonian; and 4) to summarize the many independent results that have confirmed, piecemeal, all the elements of our 4PN dynamics [16, 17].

Regarding the last item, we wish to emphasize that, besides the straightforward PN (or, equivalently, effectivefield-theory) calculations of the two-body dynamics, crucial information about the two-body dynamics has been acquired (as will be detailed below) through combining 
(in various ways) several other approaches: the effectiveone-body (EOB) formalism [22 25], the first law of binary mechanics [26 28], and gravitational self-force (SF) theory, especially when combined with the Mano-SuzukiTakasugi 29, 30] hypergeometric-expansion approach to Regge-Wheeler-Zerilli theory. We shall point out when needed the multi-way consistency checks between these approaches that have been obtained at the $4 \mathrm{PN}$ level.

Our notation for two-body systems will follow the one used in our previous works: the two masses are denoted $m_{1}, m_{2}$, and we then denote $M \equiv m_{1}+m_{2}$, $\mu \equiv m_{1} m_{2} /\left(m_{1}+m_{2}\right), \nu \equiv \mu / M=m_{1} m_{2} /\left(m_{1}+m_{2}\right)^{2}$. We indicate powers of $G$ and $c$ when it is pedagogically useful, but we sometimes set $G=c=1$ when it is more convenient.

\section{EXTANT PIECEWISE CONFIRMATIONS OF THE 4PN ADM-DELAUNAY DYNAMICS AND LIMITS ON POSSIBLE DEVIATIONS}

Let us start by recalling the structure of the $4 \mathrm{PN}$ results of Refs. 16, 17]. First, the Fokker-like reduced 2 ADM action derived in Ref. 16] has the following form (using the notation of Ref. [16]):

$$
\begin{aligned}
S_{\mathrm{DJS}}= & \int\left[\sum_{a} p_{a i} \mathrm{~d} x_{a}^{i}-H_{\mathrm{DJS}}^{\mathrm{loc}}\left(\mathbf{x}_{a}, \mathbf{p}_{a} ; s\right) \mathrm{d} t\right] \\
& +S^{\operatorname{nonloc}(s)},
\end{aligned}
$$

where the nonlocal piece of the ADM action 3 is (with $I_{i j}$ denoting the quadrupole moment of the binary system, and $\left.I_{i j}^{(3)} \equiv \mathrm{d}^{3} I_{i j} / \mathrm{d} t^{3}\right)$

$$
S^{\text {nonloc }(s)}=\frac{1}{5} \frac{G^{2} M}{c^{8}} \operatorname{Pf}_{2 s / c} \iint \frac{\mathrm{d} t \mathrm{~d} t^{\prime}}{\left|t-t^{\prime}\right|} I_{i j}^{(3)}(t) I_{i j}^{(3)}\left(t^{\prime}\right),
$$

(Pf $\equiv$ Partie finie) and where the local piece of the ADM action has the structure

$$
\begin{aligned}
H_{\mathrm{DJS}}^{\mathrm{loc}}\left(\mathbf{x}_{a}, \mathbf{p}_{a} ; s\right)= & H_{\mathrm{DJS}}^{\mathrm{loc}}\left(\mathbf{x}_{a}, \mathbf{p}_{a} ; r_{12}\right) \\
& +F\left[\mathbf{x}_{a}, \mathbf{p}_{a}\right] \ln \frac{r_{12}}{s},
\end{aligned}
$$

with

$$
F\left[\mathbf{x}_{a}, \mathbf{p}_{a}\right]=\frac{2}{5} \frac{G^{2} M}{c^{8}}\left(I_{i j}^{(3)}\right)^{2} .
$$

\footnotetext{
2 See Appendix A for a discussion of Fokker-like reduced actions.

3 In Ref. 16] the necessity of adding the nonlocal contribution $S^{\text {nonloc }(s)}$ was derived by combining the structure of the IR divergence of the local ADM action [14] with the known existence of a 4PN-level, long-range tail-transported interaction [18]. Prompted by an argument in Ref. 21], we discuss in Appendix A the (limited) extent to which $S^{\text {nonloc (s) }}$ can be (formally) directly derived from the ADM action.
}

The length scale $s$ entering the action above 4 is arbitrary because (as shown in Ref. [16]) the $s$ dependence of the nonlocal action (2.2) cancels against the $s$ dependence of the local action (2.3).

In our second paper, Ref. [17], we combined different techniques (which will be explained in detail below) for transforming the (center-of-mass-frame reduction of the) nonlocal ADM action (2.1) into an equivalent ordinary action, say

$$
S_{\mathrm{DJS}^{\prime}}=\int\left[p_{i} \mathrm{~d} q^{i}-H_{\mathrm{DJS}^{\prime}}(q, p) \mathrm{d} t\right]
$$

where $H_{\mathrm{DJS}^{\prime}}(q, p)$ is formally given by an expansion in (even) powers of $q^{i} p_{i}$. [Reference [17] gave an exact formula for the action-angle version of $S^{\text {nonloc }}$, and the explicit form of the expansion of $H_{\mathrm{DJS}^{\prime}}(q, p)$ in powers of $q^{i} p_{i}$ through $\left(q^{i} p_{i}\right)^{6}$.] The ordinary action (2.5) is equivalent to (the center-of-mass-frame reduction of) Eq. (2.1) modulo some shifts of the phase-space coordinates that are implicitly defined by the Delaunay-like reduction procedure of Ref. [17]. These shifts do not affect the gaugeinvariant observables deduced from either Eq. (2.1) or Eq. (2.5) which we shall focus on in the following. Note also in passing that the use of the EOB formalism in Ref. 17] is essentially a technical convenience, while the essential conceptual step used there is the reduction of a nonlocal action to a local form by using Delaunay-like averaging techniques (see below).

The aim of this section is to summarize the current existing confirmations of the correctness of the $4 \mathrm{PN}$ actions (2.1) or (2.5). First, we wish to recall that the full Poincaré invariance of (2.1) (in a general frame) was explicitly checked in Ref. [16] (see also Ref. [14]). This is a highly nontrivial check because the ADM derivation of the action (contrary to the harmonic-coordinates one) is far from being manifestly Lorentz invariant.

To organize the other confirmations of Eq. (2.1) or Eq. (2.5), let us note that, in the center-of-mass frame, and when using suitably scaled 5 dynamical variables [e.g. $r=$ $r^{\text {phys }} /(G M), \mathbf{p}=\mathbf{p}_{1} / \mu=-\mathbf{p}_{2} / \mu, S=S^{\text {phys }} /(G M \mu)$, $\left.\widehat{H}=\left(H-M c^{2}\right) / \mu\right]$ the rescaled Hamiltonian $\widehat{H}$ has a polynomial structure in the symmetric mass ratio $\nu$

$$
\widehat{H}=\widehat{H}_{0}+\nu \widehat{H}_{1}+\nu^{2} \widehat{H}_{2}+\nu^{3} \widehat{H}_{3}+\nu^{4} \widehat{H}_{4}
$$

where $\widehat{H}_{0}=\frac{1}{2} \mathbf{p}^{2}-1 / r+c^{-2}(\cdots)+\cdots+O\left(c^{-10}\right)$ describes the $4 \mathrm{PN}$ dynamics of a test particle in the field generated by the mass $M=m_{1}+m_{2}$.

The contribution $\nu \widehat{H}_{1}$ to the $4 \mathrm{PN}$ dynamics describes the $4 \mathrm{PN}$ approximation to the first-order self-force (1SF) dynamical effects. Over the recent years, many works

\footnotetext{
${ }^{4}$ For clarity, we do not use here the formulation where $s$ is replaced by $r_{12}=\left|\mathbf{x}_{1}-\mathbf{x}_{2}\right|$.

5 Beware that we will often oscillate between using scaled or unscaled dynamical variables.
} 
have been devoted to both the numerical and the analytical computation of $1 \mathrm{SF}$ dynamical effects. To compare these results to the predictions following from our $4 \mathrm{PN}$ dynamics (2.1) or (2.5) we need bridges between PN results and SF results. Two such PN-SF bridges have been particularly useful over the last years: the EOB formalism [22 25], and the first law of binary mechanics [26 28].

The first example of a PN-EOB-SF bridge was the derivation of the functional relation between the periastron precession of small-eccentricity orbits and the radial potentials entering the EOB Hamiltonian [6]. At the $1 \mathrm{SF}$ level, this relation [see Eqs. (5.21)-(5.25) in
6] yields the precession function $\rho(u)$ as a linear combination of $a(u), a^{\prime}(u), a^{\prime \prime}(u)$ and $\bar{d}(u)$, where $a(u)$ is the $1 \mathrm{SF}$ correction to the main EOB radial potential $A(u ; \nu)=1-2 u+\nu a(u)+O\left(\nu^{2}\right)$ [which generalizes $\left.A^{\text {Schwarzschild }}(u)=1-2 G M / c^{2} r \equiv 1-2 u\right]$, and where $\bar{d}(u)$ is the $1 \mathrm{SF}$ correction to the second EOB radial potential $\bar{D}(u) \equiv(A(u) B(u))^{-1}=1+\nu \bar{d}(u)+O\left(\nu^{2}\right)$. Let us apply this relation to the $4 \mathrm{PN}$-level values of the $\mathrm{EOB}$ potentials parametrizing the $4 \mathrm{PN}$ dynamics (2.5), as derived in Ref. [17], namely (adding the third EOB potential $\left.Q \equiv \mu^{2} \hat{Q}\right)$

$$
\begin{aligned}
A(u)= & -2 u+2 \nu u^{3}+\left(\frac{94}{3}-\frac{41 \pi^{2}}{32}\right) \nu u^{4} \\
& +\left(\left(\frac{2275 \pi^{2}}{512}-\frac{4237}{60}+\frac{128}{5} \gamma_{\mathrm{E}}+\frac{256}{5} \ln 2\right) \nu+\left(\frac{41 \pi^{2}}{32}-\frac{221}{6}\right) \nu^{2}+\frac{64}{5} \nu \ln u\right) u^{5}, \\
\bar{D}(u)= & +6 \nu u^{2}+\left(52 \nu-6 \nu^{2}\right) u^{3} \\
& +\left(\left(-\frac{533}{45}-\frac{23761 \pi^{2}}{1536}+\frac{1184}{15} \gamma_{\mathrm{E}}-\frac{6496}{15} \ln 2+\frac{2916}{5} \ln 3\right) \nu+\left(\frac{123 \pi^{2}}{16}-260\right) \nu^{2}+\frac{592}{15} \nu \ln u\right) u^{4}, \\
\hat{Q}\left(\mathbf{r}^{\prime}, \mathbf{p}^{\prime}\right)= & \left(2(4-3 \nu) \nu u^{2}+\left(\left(-\frac{5308}{15}+\frac{496256}{45} \ln 2-\frac{33048}{5} \ln 3\right) \nu-83 \nu^{2}+10 \nu^{3}\right) u^{3}\right)\left(\mathbf{n}^{\prime} \cdot \mathbf{p}^{\prime}\right)^{4} \\
& +\left(\left(-\frac{827}{3}-\frac{2358912}{25} \ln 2+\frac{1399437}{50} \ln 3+\frac{390625}{18} \ln 5\right) \nu-\frac{27}{5} \nu^{2}+6 \nu^{3}\right) u^{2}\left(\mathbf{n}^{\prime} \cdot \mathbf{p}^{\prime}\right)^{6}+O\left[\nu u\left(\mathbf{n}^{\prime} \cdot \mathbf{p}^{\prime}\right)^{8}\right] .
\end{aligned}
$$

This yields

$$
\begin{aligned}
\rho(x)=14 x^{2} & +\left(\frac{397}{2}-\frac{123}{16} \pi^{2}\right) x^{3} \\
& +\left(\frac{58265}{1536} \pi^{2}-\frac{215729}{180}+\frac{5024}{15} \gamma_{\mathrm{E}}+\frac{1184}{15} \ln 2+\frac{2916}{5} \ln 3+\frac{2512}{15} \ln x\right) x^{4}+O\left(x^{5} \ln x\right) .
\end{aligned}
$$

The $4 \mathrm{PN}$-level contribution to the precession function $\rho(x)$ is of the form $\rho_{4 \mathrm{PN}}(x)=\left(\rho_{4}^{c}+\rho_{4}^{\ln } \ln x\right) x^{4}$, with the rational logarithmic coefficient $\rho_{4}^{\ln }=\frac{2512}{15}$ [8], and the transcendental nonlogarithmic $4 \mathrm{PN}$ coefficient

$$
\begin{aligned}
\rho_{4}^{c, \text { DJS }}=\frac{58265}{1536} & \pi^{2}-\frac{215729}{180} \\
& +\frac{5024}{15} \gamma_{\mathrm{E}}+\frac{1184}{15} \ln 2+\frac{2916}{5} \ln 3 .
\end{aligned}
$$

The analytical values of the $4 \mathrm{PN}$-level functions $A, \bar{D}$, $Q$ have been recently independently confirmed by two self-force computations (based on the recently derived eccentric-extension of the first law [28]); see Refs. 31] and [32]. (Note also that the coefficients of higher powers of $\mathbf{n}^{\prime} \cdot \mathbf{p}^{\prime}$ in $Q$ were recently provided: the powers 8 and 10 in Ref. 32]; and the powers 12 to 20 in Ref. 33].)

In addition to analytical confirmations, there are also numerical self-force confirmations of the $4 \mathrm{PN}$-level values of $\rho(x)$ and $\rho_{4}^{c \text {,DJS }}$ based on direct dynamical computations of the precession of slightly eccentric orbits.

The first numerical SF determination of the precession function $\rho(u)$ was made in Ref. [34]. In particular, the latter work confirmed the value $\rho_{4}^{\ln }=\frac{2512}{15}$ and derived an estimate of the value of the nonlogarithmic coefficient, namely

$$
\rho_{4}^{c, \text { num } 34}=69_{-4}^{+7} .
$$

We were informed by Maarten van de Meent that he has 
very recently obtained a much more accurate determination of $\rho_{4}^{c}$ with preliminary results yielding [35]

$$
\rho_{4}^{c, \text { num }[35]}=64.640566(2),
$$

where the number in parentheses indicates a preliminary estimate of the uncertainty on the last digit. Note that both numerical estimates of $\rho_{4}^{c}$ assume the analytical values of the $4 \mathrm{PN}$ and $5 \mathrm{PN}$ logarithmic contributions to $\rho(u)$. [There is no doubt about the (1SF) $4 \mathrm{PN}$ and $5 \mathrm{PN}$ logarithmic contributions to the dynamics: the 4PN ones can be straightforwardly deduced from the tail-related 4PN logarithmic term written in Eq. (6.39) of Ref. [18] (equivalent to the $F\left[\mathbf{x}_{a}, \mathbf{p}_{a}\right] \ln \frac{r_{12}}{s}$ logarithmic contribution in Eq. (2.3)), while the $5 \mathrm{PN}$ ones are straightforwardly derivable from the higher-tail results of Refs. 7] and [17] (Sec. IXA).] Both numerical SF results (2.10) and (2.11) confirm, within their respective error bars, the numerical value

$$
\rho_{4}^{c, \text { DJS }}=64.6405647571193781901484255 \cdots
$$

of the result (2.9) predicted by our 4PN dynamics. The numerical result (2.10) differs from the analytical value (2.9) by

$$
\Delta \rho_{4}^{c} \equiv \rho_{4}^{c, \text { num } 34}-\rho_{4}^{c, \text { DJS }}=4_{-4}^{+7},
$$

while the more recent numerical value (2.11) differs from the analytical value (2.9) only by

$$
\Delta \rho_{4}^{c} \equiv \rho_{4}^{c, \text { num }[35}-\rho_{4}^{c, \text { DJS }}=(1 \pm 2) \times 10^{-6} .
$$

What is especially important in such a numerical check is that we are talking here about a direct dynamical check of the 4PN dynamics derived in Refs. [16] and [17]. Indeed, no use is made here of the first-law-of-mechanics bridge to go from SF computations of the Detweiler-Barack-Sago redshift invariant to $4 \mathrm{PN}$ dynamical functions. The SF computations done in Refs. 34] and 35] directly estimated the effect of the nonlocal gravitational self-force $F^{\mu}$ on slightly eccentric orbits to extract the precession function $\rho(u)$ and, then, its PN expansion coefficients. One has therefore here a direct confirmation of the way Refs. [16, 17] computed (via well-established EOB results [6]) the precession effect of the nonlocal $4 \mathrm{PN}$ dynamics.

Let us now summarize the numerical SF confirmations of the other $1 \mathrm{SF}$ predictions one can draw from the $4 \mathrm{PN}$ results (2.7). The $4 \mathrm{PN}$-level coefficients of the three EOB potentials $A, \bar{D}$ and $Q$ [the latter being considered at $O\left(p_{r}^{4}\right)$, i.e. at the level of the fourth power of the eccentricity] are of the form $A_{4 \mathrm{PN}}(u)=\nu\left(a_{5}^{c}+a_{5}^{\ln } \ln u+\nu a_{5}^{\prime}\right) u^{5}$, $\bar{D}_{4 \mathrm{PN}}(u)=\nu\left(\bar{d}_{4}^{c}+\bar{d}_{4}^{\mathrm{nn}} \ln u+\nu \bar{d}_{4}^{\prime}\right) u^{4}$, and $\hat{Q}_{4 \mathrm{PN}}\left(u, p_{r}\right)=$ $\nu q_{4,3}(\nu) u^{3} p_{r}^{4}+\nu q_{6,2}(\nu) u^{2} p_{r}^{6}+O\left(p_{r}^{8}\right)$, with $q_{4,3}(\nu)=q_{4,3}+$ $\nu q_{4,3}^{\prime}+\nu^{2} q_{4,3}^{\prime \prime}$ and $q_{6,2}(\nu)=q_{6,2}+\nu q_{6,2}^{\prime}+\nu^{2} q_{6,2}^{\prime \prime}$. Among the $4 \mathrm{PN}$ coefficients, the $1 \mathrm{SF}$ contributions are the ones at order $O(\nu)$, i.e. the unprimed ones in our notation, namely

$$
a_{5}^{c}=\frac{2275 \pi^{2}}{512}-\frac{4237}{60}+\frac{128}{5} \gamma_{\mathrm{E}}+\frac{256}{5} \ln 2,
$$

$$
\begin{aligned}
a_{5}^{\ln =} & \frac{64}{5} \\
\bar{d}_{4}^{c}= & -\frac{533}{45}-\frac{23761 \pi^{2}}{1536}+\frac{1184}{15} \gamma_{\mathrm{E}} \\
& \quad-\frac{6496}{15} \ln 2+\frac{2916}{5} \ln 3 \\
\vec{d}_{4}^{\ln }= & \frac{592}{15}, \\
q_{4,3}= & -\frac{5308}{15}+\frac{496256}{45} \ln 2-\frac{33048}{5} \ln 3, \\
q_{6,2}= & -\frac{827}{3}-\frac{2358912}{25} \ln 2 \\
& +\frac{1399437}{50} \ln 3+\frac{390625}{18} \ln 5 .
\end{aligned}
$$

The numerical values of the nonlogarithmic coefficients are

$$
\begin{aligned}
a_{5}^{c} & =23.5033892426034362387576146 \cdots, \\
\bar{d}_{4}^{c} & =221.5719911921481640232337716 \cdots, \\
q_{4,3} & =28.7110442849559497574969412 \cdots \\
q_{6,2} & =-2.7830076369522324890284545 \cdots
\end{aligned}
$$

All these 1SF-order coefficients have been independently checked, either analytically, or numerically, by various SF computations (which used the first law of binary dynamics as a bridge). We have already mentioned above the theoretical consensus on the $4 \mathrm{PN}$ logarithmic contributions $a_{5}^{\ln }$ and $\bar{d}_{4}^{\mathrm{nn}}$. [Note the absence of any logarithmic contribution to $Q_{4 \mathrm{PN}}\left(u, p_{r}\right)$.] The nonlogarithmic contribution $a_{5}^{c}$ to the main EOB radial potential $A(u)$ is actually not a deep check of the ADM results (2.1) and (2.5) because Ref. 16] used the analytical SF result of Bini and Damour [13] to calibrate their single IR ambiguity constant $C$. Let us, however, note two things. First, all the transcendental contributions to $a_{5}^{c}$ have been reproduced by the local ADM computation 12, 14], the needed calibration of $C$ using only a rational shift. Second, the analytical determination of $a_{5}^{c}$ in Ref. [13] (which was preceded by accurate numerical estimations 7, 9]) was later analytically confirmed in Refs. 36] and [37], as well as numerically confirmed by extremely high-accuracy self-force results [38].

The nonlogarithmic $1 \mathrm{SF} 4 \mathrm{PN}$ contribution $\bar{d}_{4}^{c}$ to the second EOB potential $\bar{D}(u)$ has been fully confirmed (again using the first law of mechanics) by recent SF works, both analytically and numerically. As already mentioned, direct analytical checks have been obtained in Refs. [31] and 32]. In addition, Ref. 31] has pointed out that the recent numerical SF results of Ref. [39] provide a numerical check on the value $\bar{d}_{4}^{c}$ at the accuracy level \pm 0.05 . (The latter error level takes into account the fact that the $a_{5}$ terms have been fully confirmed.)

The $1 \mathrm{SF} 4 \mathrm{PN}$ contributions to $Q\left(r, p_{r}\right)$ have also been recently confirmed, both analytically 32 and numeri- 
cally, with an uncertainty $\delta q_{4,3}= \pm 4$ [39] (see also the recent numerical determination of the coefficient $q_{4}(u)$ of $\nu p_{r}^{4}$ in the $\operatorname{EOB} Q$ potential [40]).

Let us emphasize that the terms proportional to higher powers of $\nu$ in Eq. (2.6) are much less sensitive than the terms of order $\nu$ to subtle regularization ambiguities. Actually, as shown in Refs. 10, 12 the regularization subtleties are in direct correspondence with the power of $\nu$. The terms of order $\nu^{3}$ and $\nu^{4}$ are not ambiguous at all (and have been independently derived, in the effective field theory approach, for the relation between energy and orbital frequency for circular orbits, in Ref. [11]), while the terms of order $\nu^{2}$ are delicate, but can be unambiguously derived when using dimensional regularization for treating the UV divergences [12]. Actually, as explicitly shown in Ref. 21] (see also the next section), the recent harmonic-coordinates Fokker-action computation of Ref. 21] agrees (modulo some contact transformation) with the action (2.1) for all powers of $\nu$, except for the first power. We think that this is related to the fact that the most delicate IR effects are linked with the nonlocal contribution (2.2), which is easily seen to be purely of order $\nu^{1}$ [in $S /(M \mu)$ ]. [This is also explicitly displayed in Eqs. (7.5)-(7.7) of Ref. 17].] As we shall further discuss below, physical effects mixing local and nonlocal effects, and thereby being sensitive to IR divergences, are very delicate to determine unambiguously. We can, however, conclude that the non-IR-sensitive part of the results of Ref. 21] provide an independent confirmation of all the terms in the action (2.1) which are of order $\nu^{n}$ with $n \geq 2$.

To summarize this section, many independent SF results have confirmed all the (IR-sensitive) terms linear in $\nu$, while the other $\mathrm{PN}$ calculations at the $4 \mathrm{PN}$ level (Ref. 11] and, especially, Ref. 21]), have confirmed all the terms nonlinear in $\nu$ (i.e. $\propto \nu^{2}, \nu^{3}$, and $\nu^{4}$ ). We conclude that all the results of Refs. 16, 17] have been (piecewise) confirmed.

\section{INCOMPATIBILITIES BETWEEN THE 4PN HARMONIC FOKKER ACTION RESULTS OF REF. [21] AND SELF-FORCE RESULTS}

The simplest way to compare the results of the recent harmonic Fokker action computation [21] to selfforce data is to compute the SF effects induced by the difference between the dynamics of Ref. 21], and that of Refs. [16, 17]. This difference has been worked out in Ref. [21].

Reference 21] obtained, after applying a suitable contact transformation (alluded to, though not explicitly presented, at the beginning of their Sec. V B) to their original harmonic-coordinates result an action of the same form as the ADM results (2.1), namely

$$
\begin{aligned}
S_{\mathrm{B}^{3} \mathrm{FM}}= & \int\left[\sum_{a} p_{a i} \mathrm{~d} x_{a}^{i}-H_{\mathrm{B}^{3} \mathrm{FM}}^{\mathrm{loc}}\left(\mathbf{x}_{a}, \mathbf{p}_{a} ; s\right) \mathrm{d} t\right] \\
& +S^{\operatorname{nonloc}(s)}
\end{aligned}
$$

with the same nonlocal (or "tail") action6, but with a different local Hamiltonian,

$$
\begin{aligned}
H_{\mathrm{B}^{3} \mathrm{FM}}^{\mathrm{loc}}\left(\mathbf{x}_{a}, \mathbf{p}_{a} ; s\right)= & H_{\mathrm{B}^{3} \mathrm{FM}}^{\mathrm{loc}}\left(\mathbf{x}_{a}, \mathbf{p}_{a} ; r_{12}\right) \\
& +F\left[\mathbf{x}_{a}, \mathbf{p}_{a}\right] \ln \frac{r_{12}}{s} .
\end{aligned}
$$

(Reference 21] does not explicitly display the ln $s$ dependence of the local Hamiltonian but agrees with Ref. [16] on the cancellation of that dependence.) For simplicity, we do not discuss here the issue of the order reduction of the derivatives of $\mathbf{x}_{a}$ and $\mathbf{p}_{a}$ entering (via $I_{i j}^{(3)}$ ) both the nonlocal action (2.2) and the coefficient $F$ of the logarithm, Eq. (2.4). Indeed, there is complete agreement, at the level of the action, between Refs. [16] and [21] for what concerns the nonlocal piece of the action. [As said in Ref. [16], the order reduction of $I_{i j}^{(3)}$ (i.e. its on-shell replacement by a local function of $\mathbf{x}_{a}(t)$ and $\left.\mathbf{p}_{a}(t)\right)$ entails a suitable nonlocal shift of the dynamical variables (which was explicated in Eqs. (5.14), (5.15) of Ref. [21]).] The important issue is the difference between the two local Hamiltonians which, according to Eq. (5.19) of Ref. [21], is

$$
\begin{aligned}
H_{\mathrm{B}^{3} \mathrm{FM}}^{\mathrm{loc}}-H_{\mathrm{DJS}}^{\mathrm{loc}}= & \frac{G^{4} M m_{1}^{2} m_{2}^{2}}{c^{8} r_{12}^{4}} \\
& \times\left[a_{\mathrm{B}^{3} \mathrm{FM}}\left(\frac{\left(\mathbf{n}_{12} \cdot \mathbf{p}_{1}\right)}{m_{1}}-\frac{\left(\mathbf{n}_{12} \cdot \mathbf{p}_{2}\right)}{m_{2}}\right)^{2}\right. \\
& \left.+b_{\mathrm{B}^{3} \mathrm{FM}}\left(\frac{\mathbf{p}_{1}}{m_{1}}-\frac{\mathbf{p}_{2}}{m_{2}}\right)^{2}+c_{\mathrm{B}^{3} \mathrm{FM}} \frac{G M}{r_{12}}\right],
\end{aligned}
$$

with

$$
(a, b, c)_{\mathrm{B}^{3} \mathrm{FM}}=\left(\frac{1429}{315}, \frac{826}{315}, \frac{902}{315}\right) .
$$

Before discussing further the origin of the discrepancy [i.e. the fact that $\left.(a, b, c)_{\mathrm{B}^{3} \mathrm{FM}} \neq(0,0,0)\right]$, we wish to emphasize two things: (i) the discrepant terms in Eq. (3.3) represent only three (Galileo-invariant) terms among the hundreds (exactly 219) of contributions to the (noncenter-of-mass) two-body Hamiltonian, as displayed in the Appendix of Ref. [16], or in Sec. VIII E of Ref. [14]; (ii) the three discrepant terms (3.3) [when expressed in the center of mass, and in reduced variables, i.e. in the sense of Eq. (2.6)] are linear in the symmetric mass ratio $\nu$, i.e. they are of $1 \mathrm{SF}$ order.

The discrepancy Eq. (3.3) therefore implies 1SFdetectable effects away from all the 1SF checks of the ADM dynamics (2.1) reviewed in the previous section.

\footnotetext{
6 We use here the fact that, at the $4 \mathrm{PN}$ level, $M_{\mathrm{B}^{3} \mathrm{FM}}=M_{\mathrm{DJS}}+$ $O\left(1 / c^{2}\right)$ with $M_{\mathrm{DJS}} \equiv M^{\text {here }}=m_{1}+m_{2}$.
} 
Let us now quantify the corresponding $1 \mathrm{SF}$ differences. To do that it is convenient to transcribe the $4 \mathrm{PN}$-level Hamiltonian difference 3.3 in terms of corresponding differences in the three EOB potentials $A, \bar{D}, Q$. (We recall in passing that the EOB parametrization of the dynamics in terms of $A, \bar{D}, Q$ is completely gauge fixed, and therefore directly linked to gauge-invariant quantities.)

Considering, for more generality, a 4PN-level Hamiltonian difference with general coefficients $(a, b, c)$ in Eq. (3.3), the corresponding additional contributions to $A$, $\bar{D}, Q$ are easily found to be

$$
\begin{aligned}
& \delta^{a, b, c} A=(2 b+2 c) \nu u^{5}, \\
& \delta^{a, b, c} \bar{D}=(2 a+8 b) \nu u^{4}, \\
& \delta^{a, b, c} Q=0 .
\end{aligned}
$$

Note in passing that those contributions are invariant under the "gauge transformation"

$$
\delta^{g}(a, b, c)=g(4,-1,1),
$$

which corresponds to the most general canonical transformation respecting the structure (3.3) (with generating function $\left.\propto g \nu p_{r} / r^{3}\right)$. Inserting the values (3.4) in Eqs. (3.5a) -3.5b) leads to changes in $A$ and $\bar{D}$ (with respect to the values computed in Ref. [17])

$$
\begin{aligned}
\delta_{4 \mathrm{PN}}^{\mathrm{B}^{3} \mathrm{FM}} A & =\frac{384}{35} \nu u^{5}, \\
\delta_{4 \mathrm{PN}}^{\mathrm{B}^{3} \mathrm{FM}} \bar{D} & =\frac{9466}{315} \nu u^{4} .
\end{aligned}
$$

In other words, the only $1 \mathrm{SF} 4 \mathrm{PN}$-level coefficients that are different are $a_{5}^{c}$ and $\bar{d}_{4}^{c}$ with

$$
\begin{aligned}
& \delta^{\mathrm{B}^{3} \mathrm{FM}} a_{5}^{c}=\frac{384}{35} \approx 10.97143 \\
& \delta^{\mathrm{B}^{3} \mathrm{FM}} \bar{d}_{4}^{c}=\frac{9466}{315} \approx 30.05079 .
\end{aligned}
$$

Such large, 4PN-level deviations away from the results of Ref. [17] are in violent contradiction with the many $\mathrm{SF}$ confirmations of the ADM 4PN dynamics reviewed in the previous section. They have been obtained here by using the Delaunay-like reduction used in Ref. [17] to convert the nonlocal dynamics (2.1) into the (formally) local one (2.5). The authors of Ref. 21] express doubts about some aspects of the results of [17]. We shall address their concerns in the following sections and conclude that their concerns are unsubstantiated. Therefore we conclude that taking the results of Ref. [21] at face value does lead to the large changes (3.9) and (3.10) above, and are therefore strictly incompatible with extant SF knowledge.

We wish to go further and point out an even more blatant contradiction with SF tests of periapsis precession. First, we note (using, e.g. Eq. (8.3) in [17]) that the changes above entail a corresponding change in the $4 \mathrm{PN}-$ level precession coefficient $\rho_{4}^{c}$ given by $\delta \rho_{4}^{c}=10 \delta a_{5}^{c}+\delta \bar{d}_{4}^{c}$, i.e.

$$
\delta^{\mathrm{B}^{3} \mathrm{FM}} \rho_{4}^{c}=(2 a+28 b+20 c)_{\mathrm{B}^{3} \mathrm{FM}},
$$

i.e.

$$
\delta^{\mathrm{B}^{3} \mathrm{FM}} \rho_{4}^{c}=\frac{44026}{315} \approx 139.7650794 .
$$

As we recalled above, SF computations [34, 35] of the precession function $\rho(u)$ [6], and of its PN coefficients, do not rely on the first law of binary mechanics, but involve direct computations of the additional precession induced by the nonlocal self-force. The difference (3.12) is therefore (assuming the correctness of the Delaunay reduction of Ref. 17] ) a direct dynamical consequence of the Fokker-action result of Ref. 21]. It would be interesting to confirm this result by a purely dynamical computation of the (nonlocal) 4PN precession. We note here that the difference is already excluded by the "old" result (2.13), and even more so (by $10^{8}$ standard deviations!) by the recent one 2.14).

\section{LOGICAL BASIS OF THE ACTION-ANGLE DELAUNAY-LIKE METHOD OF REF. [17]}

Independently of the above SF confirmations of the 4PN results of Refs. [16, 17], let us reassess the logical basis and the consistency of the methodology used in our Delaunay-EOB derivation, and indicate, by contrast, where, in our opinion, lie the flaws of Ref. 21] that have led to the nonzero values (3.4), which are incompatible with many SF results. Our Ref. framework is the action-angle formulation of (planar) Hamiltonian dynamics. In [17] we used the standard Delaunay notation (modulo the use of calligraphic letters $L \rightarrow \mathcal{L}$, $G \rightarrow \mathcal{G})$. Namely, $(\mathcal{L}, \ell ; \mathcal{G}, g)$ are the two planar actionangle canonical pairs: $\mathcal{L}=\sqrt{a}$ is conjugate to the mean anomaly angle $\ell$, while $\mathcal{G}=\sqrt{a\left(1-e^{2}\right)}$ is conjugate to the argument of the periastron $g=\omega$. In order to better exhibit the meaning (and consistency) of our approach in the circular limit, we shall use here the combination of Delaunay variables introduced by Poincaré, namely, the two action-angle pairs $\left(\Lambda, \lambda ; I_{r}, \varpi\right)$ where (using suitably scaled variables as in Ref. [17])

$$
\begin{aligned}
\Lambda & =\mathcal{L}=I_{r}+I_{\varphi}, \\
\lambda & =\ell+g, \\
I_{r} & =\mathcal{L}-\mathcal{G}=\mathcal{L}-I_{\varphi}, \\
\varpi & =-g=-\omega .
\end{aligned}
$$

Here, $I_{\varphi}=\mathcal{G}$ is the angular momentum $\left(I_{\varphi}=\right.$ $\left.\frac{1}{2 \pi} \oint p_{\varphi} \mathrm{d} \varphi=p_{\varphi}\right)$ and $I_{r}$ is the radial action $\left(I_{r}=\right.$ $\left.\frac{1}{2 \pi} \oint p_{r} \mathrm{~d} r\right)$. The sum $\Lambda=I_{r}+I_{\varphi}$ is conjugate to the mean longitude $\lambda=\ell+g$, while $I_{r}$ is conjugate 
to (minus) the argument of the periastron $\varpi=-\omega$. The latter (surprising) minus sign is necessary to have $\mathrm{d} L \wedge \mathrm{d} \ell+\mathrm{d} G \wedge \mathrm{d} g=\mathrm{d} \Lambda \wedge \mathrm{d} \lambda+\mathrm{d} I_{r} \wedge \mathrm{d} \varpi$ with $I_{r}=$ $\mathcal{L}-\mathcal{G}=\sqrt{a}-\sqrt{a\left(1-e^{2}\right)}>0$. In the circular limit, $\lambda$ becomes the usual polar angle $\varphi$ in the orbital plane and $I_{r} \rightarrow 0$, so that the action variable $\Lambda$ becomes equal to the angular momentum $I_{\varphi}=p_{\varphi}$. In this limit, the general Hamilton equation $\dot{\lambda}=\partial H / \partial \Lambda$ gives back the usual circular link between the orbital frequency $\Omega=\dot{\varphi}$ and the derivative of the energy with respect to the angular momentum $p_{\varphi}$. However, when dealing with noncircular orbits, and tackling the nonlocal action (2.2), it is important to work with the clearly defined canonical action-angle pairs $\left(\Lambda, \lambda ; I_{r}, \varpi\right)$. In principle, it would be better, when discussing the circular limit, to replace the second pair $\left(I_{r}, \varpi\right)$ by the associated Poincaré variables $\xi=\sqrt{2 I_{r}} \cos \varpi, \eta=\sqrt{2 I_{r}} \sin \varpi$, because they are canonical $\left(\mathrm{d} \xi \wedge \mathrm{d} \eta=\mathrm{d} I_{r} \wedge \mathrm{d} \varpi\right)$ and regular in the circular limit (while $\varpi$ becomes ill defined as $I_{r} \rightarrow 0$ ). Keeping in mind such an additional change of variables, we shall, however, find simpler to express our methodology in terms of $I_{r}$ and $\varpi$.

The basic methodology we used in Ref. [17] for dealing with the nonlocal 4PN dynamics, Eqs. 2.1 and (2.2), consists of four steps: (a) we reexpress the action (2.1) as a nonlocal action in the action-angle variables $\left(\Lambda, \lambda ; I_{r}, \varpi\right)$; (b) we expand it (formally to infinite order) in powers of the eccentricity, i.e. in powers of $\sqrt{I_{r}}$; (c) we "order reduce" the nonlocal dependence on the action-angle variables by using the on-shell equations of motion7; and (d) we eliminate, à la Delaunay, the periodic terms in the order-reduced Hamiltonian by a canonical transformation of the action-angle variables. After these four steps, we end up with an Hamiltonian which is an ordinary (local) function of the (transformed) action variables alone.

In order not to get distracted by irrelevant technicalities, let us illustrate this methodology [which was applied in Ref. [17] to the full 4PN action (2.1) - (2.2)] on a simpler toy example which contains some of the key ingredients of the action (2.1)-(2.2), namely the action

$$
\begin{aligned}
S_{\mathrm{toy}}= & \int\left[\mathbf{p}(t) \cdot \mathrm{d} \mathbf{r}(t)-H_{\mathrm{toy}}(t) \mathrm{d} t\right], \\
H_{\mathrm{toy}}(t)= & \frac{1}{2} \mathbf{p}(t)^{2}-\frac{1}{r(t)} \\
& +\varepsilon \int_{-\infty}^{+\infty} \mathrm{d} t^{\prime} \mu\left(t^{\prime}-t\right) \frac{\mathbf{r}\left(t^{\prime}\right) \cdot \mathbf{r}(t)}{\left|\mathbf{r}\left(t^{\prime}\right)\right|^{3}|\mathbf{r}(t)|^{3}}
\end{aligned}
$$

where $\mu(\tau)$ is an even function of the time difference $\tau \equiv t^{\prime}-t$. In the real case (2.1)-(2.2), $\mu(\tau)=1 /|\tau|$ with an additional partie finie (Pf) prescription. The

\footnotetext{
7 As discussed in detail below, this is equivalent to applying suitable nonlocal shifts of the phase-space variables.
}

subtleties linked to the Pf prescription and to the slow decay of $1 /|\tau|$ for $|\tau| \rightarrow+\infty$ are only of a technical nature. For instance, the oscillatory nature of the integrand $I_{i j}^{\prime(3)} I_{i j}^{(3)}$ [mimicked as $x_{i}^{\prime} x_{i} /\left(r^{\prime 3} r^{3}\right)$ in our toy example] ensures the large $|\tau|$ convergence 8 of the nonlocal integral (2.2) without having to assume that $I_{i j}^{(3)}$ tends to zero when $|\tau| \rightarrow+\infty$. [Indeed, the fact that $-x_{i} / r^{3}$ is the on-shell value of the second time derivative of $x_{i}$, and $I_{i j}^{(3)}$ is the third time derivative of $I_{i j}$, ensures that their large-time averages both vanish for conservative bound motions. Note that our toy model is the "electromagnetic" (dipolar) analog of the gravitational (quadrupolar) tail action.] For simplicity, as we wish here to emphasize issues of principle without getting bogged down by secondary technical issues, we will not specify the weight $\mu(\tau)$ used in our toy model, but proceed as if it were a smooth, integrable even function of $\tau$.

The first two steps, (a) and (b), of our procedure yield (when considered, for illustration, at linear order in the eccentricity $e$ )

$$
\begin{aligned}
H_{\text {toy }}(t)= & -\frac{1}{2 \Lambda^{2}}+\varepsilon \int_{-\infty}^{+\infty} \mathrm{d} \tau \mu(\tau) \frac{1}{\Lambda^{4} \Lambda^{\prime 4}}\left[\cos \left(\lambda^{\prime}-\lambda\right)\right. \\
& +2 e \cos \left(2 \lambda-\lambda^{\prime}+\varpi\right)+2 e^{\prime} \cos \left(2 \lambda^{\prime}-\lambda+\varpi^{\prime}\right) \\
& \left.+O\left(e^{2}\right)\right] .
\end{aligned}
$$

Here and below $f^{\prime}$ denotes the variable $f$ taken at time $t^{\prime}=t+\tau$, while $f$ denotes its value at time $t$.

Step (c) consists in writing, for any variable $f$, its value $f^{\prime}$ at the shifted time $t^{\prime}$ in terms of canonical variables at time $t$ and of an integral over intermediate times involving the rhs of the (Hamiltonian) equations of motion. For a nonlocal action, the latter read $S_{\lambda}(t)=0, S_{\Lambda}(t)=0$, etc., with

$$
\begin{aligned}
& S_{\lambda}(t) \equiv \frac{\delta S}{\delta \lambda(t)} \equiv-\dot{\Lambda}(t)-\frac{\delta H}{\delta \lambda(t)}, \\
& S_{\Lambda}(t) \equiv \frac{\delta S}{\delta \Lambda(t)} \equiv \dot{\lambda}(t)-\frac{\delta H}{\delta \Lambda(t)}, \quad \text { etc. }
\end{aligned}
$$

where $\delta / \delta f(t)$ denotes a functional derivative [acting on the action $S$ or on $\left.\int \mathrm{d} t H^{\text {nonlocal }}(t)\right]$. In the normal case of local actions [41, 42], the use of the identities 44.5a) and $4.5 \mathrm{~b}$ ) is the main tool allowing one to show how the replacement of the equations of motion within an action is equivalent to a suitable shift of the dynamical variables (a "field redefinition"). In the case of nonlocal actions, one must integrate the identities (4.5a) and 4.5b before replacing $\lambda^{\prime} \equiv \lambda(t+\tau), \Lambda^{\prime} \equiv \Lambda(t+\tau), \cdots$ in the nonlocal piece of the action. In this integration, the terms

\footnotetext{
8 We have convergence both at $\tau \rightarrow+\infty$ and $\tau \rightarrow-\infty$, without having absolute convergence. See Appendix A for more discussion of convergence issues in reduced actions.
} 
$S_{\lambda}(t), S_{\Lambda}(t), \cdots$ are treated as additional terms, which would be zero on shell, but which are now considered as "source terms" on the rhs of the usual Hamilton equations of motion, $\dot{\lambda}(t)-\delta H / \delta \Lambda(t)=S_{\Lambda}, \cdots$. In view of the well-known possibility of neglecting "double-zero terms," it is enough to work linearly in these (simplezero) source terms. Moreover, as we are working within a PN-expanded scheme (and as the nonlocality enters only at order $\left.\varepsilon=1 / c^{8}\right)$, it is actually enough [modulo terms of order $O\left(c^{-2} \varepsilon\right)$ ], for the purpose of replacing $\lambda^{\prime}, \Lambda^{\prime}, \cdots$ in the nonlocal piece of the action, to compute them as solutions of the (sourced) Newtonian-level equations of motion, i.e. solutions of the differential equations (4.5a) and 4.5b) with $\delta H / \delta \Lambda, \cdots \rightarrow \delta H_{0} / \delta \Lambda, \cdots$, where $H_{0}=-1 /\left(2 \Lambda^{2}\right)$. The explicit form of these sourced equations of motion are

$$
\begin{aligned}
& \dot{\Lambda}(t)=-S_{\lambda}(t), \\
& \dot{\lambda}(t)=\frac{1}{\Lambda^{3}(t)}+S_{\Lambda}(t), \quad \text { etc. }
\end{aligned}
$$

Viewing these equations as differential equations with respect to $t^{\prime}=t+\tau$ (or $\tau$ ) for the unknowns $\Lambda^{\prime} \equiv \Lambda\left(t^{\prime}\right)$ and $\lambda^{\prime} \equiv \lambda\left(t^{\prime}\right)$, and imposing the initial conditions at $t^{\prime}=t$ (i.e. $\tau=0), \Lambda^{\prime}\left(t^{\prime}=t\right)=\Lambda, \lambda^{\prime}\left(t^{\prime}=t\right)=\lambda$, yields as a unique solution (to linear order in the source terms)

$$
\begin{aligned}
\Lambda^{\prime}= & \Lambda-\int_{t}^{t^{\prime}} \mathrm{d} t_{1} S_{\lambda}\left(t_{1}\right) \\
\lambda^{\prime}= & \lambda+\frac{t^{\prime}-t}{\Lambda^{3}}+\frac{3}{\Lambda^{4}} \int_{t}^{t^{\prime}} \mathrm{d} t_{1}\left(t^{\prime}-t_{1}\right) S_{\lambda}\left(t_{1}\right) \\
& +\int_{t}^{t^{\prime}} \mathrm{d} t_{1} S_{\Lambda}\left(t_{1}\right) .
\end{aligned}
$$

(We do not assume here $t<t^{\prime}$ but use the convention that $\int_{t}^{t^{\prime}}=-\int_{t^{\prime}}^{t}$ ) When replacing the identities (4.7) in a nonlocal action $S=\int \mathrm{d} t \mathrm{~d} t^{\prime} \mu\left(t^{\prime}-t\right) \mathcal{S}\left(\Lambda, \Lambda^{\prime}, \lambda^{\prime}-\right.$ $\lambda, \cdots$ ) (keeping only the terms linear in the source terms, which is allowed modulo "double-zero" terms), the extra terms involving the source contributions will have the form $\int \mathrm{d} t_{1}\left[\xi_{\Lambda}\left(t_{1}\right) S_{\Lambda}\left(t_{1}\right)+\xi_{\lambda}\left(t_{1}\right) S_{\lambda}\left(t_{1}\right)+\cdots\right]$, where the quantities $\xi_{\Lambda}\left(t_{1}\right), \xi_{\lambda}\left(t_{1}\right), \cdots$ are given by integrals over $t$ and $t^{\prime}$ of a function of dynamical variables: $\xi_{\Lambda}\left(t_{1}\right)=\iint \mathrm{d} t \mathrm{~d} t^{\prime} \mu\left(t^{\prime}-t\right) \mathcal{A}\left(t^{\prime}-t, t_{1}-t, \Lambda(t), \lambda(t)\right), \cdots$. (Note that, because of the inequalities $t<t_{1}<t^{\prime}$ or $t^{\prime}<t_{1}<t$, for given values of $t_{1}$ and $\tau=t^{\prime}-t, t$ and $t^{\prime}$ both range over a bounded interval.) The extra terms involving the source contributions can then be "field-redefined away" by corresponding shifts of the dynamical variables: $\delta \Lambda\left(t_{1}\right)=\xi_{\Lambda}\left(t_{1}\right), \delta \lambda\left(t_{1}\right)=\xi_{\lambda}\left(t_{1}\right), \cdots$. [These shifts have, in general, nonlocal structures of the type $\delta \Lambda\left(t_{1}\right)=\int \mathrm{d} t K_{\Lambda}\left(t-t_{1}, \Lambda(t), \lambda(t)\right), \delta \lambda\left(t_{1}\right)=$ $\int \mathrm{d} t K_{\lambda}\left(t-t_{1}, \Lambda(t), \lambda(t)\right)$, etc. $]$

After performing these (nonlocal) shifts, we are left with an action obtained by replacing $\lambda^{\prime}, \Lambda^{\prime}, \cdots$ by the rhs of Eq. (4.7) in which one has set to zero the terms
$S_{\lambda}\left(t_{1}\right), S_{\Lambda}\left(t_{1}\right), \cdots$. In other words, the use of these shifts justifies the naive replacement of $\lambda^{\prime}, \Lambda^{\prime}, \cdots$ by the solution of the (Newtonian) equations of motion, namely

$$
\begin{aligned}
& \lambda^{\prime}=\lambda+\frac{\tau}{\Lambda^{3}}, \\
& \Lambda^{\prime}=\Lambda .
\end{aligned}
$$

Note that the very simple time structure of the unperturbed solution in action-angle variables plays a very useful role in our Delaunay-based reduction procedure.

With the replacements (4.8a) and 4.8b [i.e. after steps (a), (b), and (c)] we have a Hamiltonian of the form

$$
\begin{aligned}
H_{\text {toy }}^{\prime}(t)= & -\frac{1}{2 \Lambda^{2}}+\varepsilon \int_{-\infty}^{+\infty} \mathrm{d} \tau \mu(\tau) \frac{1}{\Lambda^{8}}\left\{\cos \left(\frac{\tau}{\Lambda^{3}}\right)\right. \\
& +2 e\left[\cos \left(\frac{\tau}{\Lambda^{3}}-\lambda-\varpi\right)\right. \\
& \left.\left.+\cos \left(\frac{2 \tau}{\Lambda^{3}}+\lambda+\varpi\right)\right]+O\left(e^{2}\right)\right\}
\end{aligned}
$$

where we recall that $e$ is the function of $I_{r} / \Lambda$ defined so that $1-\sqrt{1-e^{2}}=I_{r} / \Lambda$, i.e.

$$
e=\sqrt{1-\left(1-\frac{I_{r}}{\Lambda}\right)^{2}}
$$

At this stage, Eq. (4.9) defines an ordinary Hamiltonian, expressed in terms of action-angle variables $\left(\Lambda, \lambda ; I_{r}, \varpi\right)$. [Strictly speaking, the variables $\Lambda, \lambda$, etc., at the stage of Eq. (4.9) differ from the ones entering the original action by the shifts $\xi_{\Lambda}, \xi_{\lambda}$, etc., mentioned above. They should be denoted as $\Lambda^{\text {shifted }}, \lambda^{\text {shifted }}$, etc., but, for simplicity, we do not indicate the shifted nature of $\Lambda, \lambda, \ldots$.

As we are now dealing with an ordinary, local Hamiltonian, our fourth step (d), i.e. the Delaunay elimination of periodic terms by suitable canonical transformations is standard. For instance, a generating function proportional to

$$
\varepsilon \int_{-\infty}^{+\infty} \mathrm{d} \tau \mu(\tau) \frac{1}{\Lambda^{5}} \sin \left(\frac{\tau}{\Lambda^{3}}-\lambda-\varpi\right)
$$

will eliminate the contribution $+2 e \cos \left(\tau / \Lambda^{3}-\lambda-\varpi\right)$ on the rhs of Eq. (4.9).

After completing step (d) (formally to all orders in the eccentricity) we end up with an ordinary (local) Hamiltonian that depends only on (shifted) action variables. E.g., for our toy model

$$
\begin{aligned}
H_{\text {toy }}^{\prime \prime}\left(\Lambda, I_{r}\right)=- & \frac{1}{2 \Lambda^{2}}+\varepsilon \int_{-\infty}^{+\infty} \mathrm{d} \tau \mu(\tau) \frac{1}{\Lambda^{8}} \\
& \times\left[\cos \left(\frac{\tau}{\Lambda^{3}}\right)+O\left(e^{2}\right)\right]
\end{aligned}
$$


Let us emphasize that our procedure allows one, in particular, to construct (formally to all orders in eccentricity) a strictly conserved energy that consistently includes the nonlocal tails at the $4 \mathrm{PN}$ level. [The nonlocal effects are recognizable through their integral nature, e.g. the integral $\varepsilon \int_{-\infty}^{+\infty} \mathrm{d} \tau \mu(\tau)(\cdots)$ in Eq. (4.12).] Namely, our final, Delaunay Hamiltonian is strictly conserved. This shows that the contrary statement below Eq. (5.17) in Ref. 21] is incorrect (at least when allowing for an expansion in powers of $\left.I_{r} / \Lambda \sim e^{2}\right)$.

In addition, our construction also gives two conserved action variables: 9: $\Lambda$ and $I_{r}$. These conserved quantities are such that the difference, $\Lambda-I_{r}$ is conserved and reduces to the usual orbital angular momentum $I_{\varphi}=\frac{1}{2 \pi} \oint p_{\varphi} \mathrm{d} \varphi=p_{\varphi}$ in the local case. We can then consider that $I_{\varphi}:=\Lambda-I_{r}$ defines a strictly conserved angular momentum that consistently includes the $4 \mathrm{PN}$ nonlocal dynamical effects. (Indeed, one easily checks that the rotational symmetry $\varphi^{\prime}=\varphi+$ const remains a symmetry at all stages of our construction so that $I_{\varphi}$ is indeed the Noether conserved quantity associated with this symmetry.)

Let us also emphasize another consistency feature of our final (reduced, local, Delaunay) Hamiltonian. As the above detailed derivation clearly shows, our final Hamiltonian action (in terms of $\Lambda^{\text {shifted }}, \lambda^{\text {shifted }}, \ldots$ but without explicitly indicating the shifts) is simply

$$
S^{\prime \prime}=\int\left[\Lambda \mathrm{d} \lambda+I_{r} \mathrm{~d} \varpi-H^{\prime \prime}\left(\Lambda, I_{r}\right) \mathrm{d} t\right]
$$

and the corresponding equations of motion are the usual ones:

$$
\dot{\lambda}=\frac{\partial H^{\prime \prime}\left(\Lambda, I_{r}\right)}{\partial \Lambda}, \quad \dot{\varpi}=\frac{\partial H^{\prime \prime}\left(\Lambda, I_{r}\right)}{\partial I_{r}}, \quad \dot{\Lambda}=0, \quad \dot{I}_{r}=0 .
$$

We said above that, in the circular limit, the mean anomaly $\lambda=\ell+g=\ell+\omega$ reduces to a usual polar angle $\varphi$. (Indeed, this is true in the Newtonian case, and, as all the Delaunay shifts are periodic in the unique fast angular variable $\lambda$, we have that $\varphi=\lambda+$ terms periodic in $\lambda$ that vanish with $e$.) Therefore our derivation shows, among other things, that the orbital frequency $\Omega(=\dot{\lambda})$ of a circular $\left(I_{r}=0\right)$ binary (with conserved angular momentum $\Lambda=I_{\varphi}$ ) is given by the usual formula

$$
\Omega=\dot{\lambda}=\left.\frac{\partial H^{\prime \prime}\left(\Lambda, I_{r}\right)}{\partial \Lambda}\right|_{I_{r}=0}=\left.\frac{\partial H^{\prime \prime}\left(I_{r}+I_{\varphi}, I_{r}\right)}{\partial I_{\varphi}}\right|_{I_{r}=0} .
$$

In particular, this means, in our toy example, that the circular angular frequency is of the form

$$
\Omega_{\mathrm{toy}}=\frac{\partial H_{\mathrm{toy}}^{\prime \prime}(\Lambda, 0)}{\partial \Lambda}
$$

9 It would be interesting to study whether our (4PN-order) construction of conserved action variables, and conserved energy, is related to the (1SF-order) work of Ref. [43].

$$
=\frac{1}{\Lambda^{3}}+\varepsilon \frac{\partial}{\partial \Lambda}\left(\int_{-\infty}^{+\infty} \mathrm{d} \tau \mu(\tau) \frac{1}{\Lambda^{8}} \cos \left(\frac{\tau}{\Lambda^{3}}\right)\right) .
$$

One sees that the computation of the rhs will involve two types of contributions coming from the tail term: a "normal" contribution where the $\Lambda$ differentiation of the second term acts on the prefactor $1 / \Lambda^{8}$ of the tail integral, and a "new" contribution where $\partial / \partial \Lambda$ acts on the argument of $\cos \left(\tau / \Lambda^{3}\right)$.

We recall that, in the full $4 \mathrm{PN}$ case, the analog of our final result (4.12) [say, for simplicity, at the $O\left(e^{0}\right)$ level] is the one discussed in Sec. V of Ref. [16], which leads to (with $J$ now denoting $I_{\varphi} \equiv \Lambda-I_{r}$ )

$$
\begin{aligned}
E_{4 \mathrm{PN}}^{\mathrm{circ}}(J)= & H_{\mathrm{DJS}}^{\mathrm{loc}}(\mathbf{r}, \mathbf{p} ; s)+F(\mathbf{r}, \mathbf{p}) \ln \frac{r}{s} \\
& -\frac{1}{5} \frac{G^{2} M}{c^{8}} \operatorname{Pf}_{2 s / c} \int_{-\infty}^{+\infty} \frac{\mathrm{d} \tau}{|\tau|} f(\tau),
\end{aligned}
$$

where $f(\tau):=\left[I_{i j}^{(3)}(t+\tau) I_{i j}^{(3)}(t)\right]^{\text {circ }}$, as in Sec. V of Ref. [16]. [In the circular limit $f(\tau)$ does not depend on $t$.] The explicit value of the last, tail term 10 is given, in view of Eqs. (5.7)-(5.9) in Ref. [16] by

$$
\begin{aligned}
{\left[H^{\text {tail }(s)}\right]^{\text {circ }}(J) } & =\frac{64}{5} \frac{G^{2} M}{c^{8}}\left(\mu \Omega^{3} r_{12}^{2}\right)^{2} \ln \left(4 \mathrm{e}^{\gamma_{\mathrm{E}}} \frac{\Omega(J) s}{c}\right) \\
& =\frac{64}{5} \mu c^{2} \frac{\nu}{j^{10}} \ln \left(\frac{4 \mathrm{e}^{\gamma_{\mathrm{E}}} \hat{s}}{j^{3}}\right)
\end{aligned}
$$

where $j \equiv c J /(G M \mu)$, and $\hat{s} \equiv s / m$ with $m \equiv G M / c^{2}$.

If we alternatively decide to define the tail contribution to the reduced Hamiltonian by incorporating the term $F \ln (r / s)$ in it, we must simply replace the scale $s$ by $r=r_{12}$, so that (using the fact that along Newtonian circular orbits $\left.r^{\text {circ }}=m j^{2}\right)$

$$
\left[H^{\text {tail }\left(s=r_{12}\right)}\right]^{\operatorname{circ}}(J)=\frac{64}{5} \mu c^{2} \frac{\nu}{j^{10}} \ln \left(\frac{4 \mathrm{e}^{\gamma_{\mathrm{E}}}}{j}\right) .
$$

Evidently, we must keep in mind that the meaning of the two quantities (4.18) and (4.19) is different and that their $J$ derivative will differ by a term involving the $J$ derivative of $r^{\text {circ }}=m j^{2}$. But the latter difference has a conceptually trivial origin [in view of the local nature of the $F \ln (r / s)$ term in the Hamiltonian] and does not interfere with the issue we wish to emphasize here, namely the existence, in the orbital frequency, $\Omega^{\text {circ }}=\mathrm{d} H^{\text {circ }} / \mathrm{d} J$, of a contribution coming from differentiating the $J$ dependence of the argument $4 \mathrm{e}^{\gamma_{\mathrm{E}}} \Omega(J) s / c$ of the logarithm in the first (or second) line of Eq. (4.18).

10 Here, we find it convenient to work with a tail term defined with some given, constant scale $s$. Such a scale is not a dynamical variable and is not affected by the $\Lambda$ differentiation above, i.e. the $f$ differentiation in the present circular case. 
As clearly pointed out already in Ref. 16], the computation of the tail contribution to the (circular, Delaunay) function $H(J)=E(J)$ "involves the evaluation of the nonlocal piece $\left[H_{4 \mathrm{PN}}^{\text {tail }(s)}\right]$ along circular motion (without any differentiation)". This lack of any differentiation in the evaluation of $\left[H^{\text {tail }}\right]^{\text {circ }}(J)$ distinguishes it among the other gauge-invariant functions one can associate with the sequence of circular orbits, and rendered very clear 11 that this necessarily led to the correct value of the function $\left[H^{\text {tail }}\right]^{\text {circ }}(J)$. Our discussion above has confirmed the consistency of this result and has shown that, indeed, when computing the orbital frequency it is correct to $J$ differentiate the argument of the tail log.

Let us now derive the tail-log contribution to the functional relation between energy and orbital frequency, $E(\Omega)$, corresponding to Eq. 4.19).

We start by deriving a useful general result about the function $E(\Omega)$, or equivalently the function $E(x)$ [where $\left.x \equiv\left(G M \Omega / c^{3}\right)^{2 / 3}\right]$. Let us first consider the case of an ordinary Hamiltonian dynamics of the type (with $J \equiv$ $\left.p_{\varphi}\right)$

$$
H^{\mathrm{ord}}\left(r, p_{r}, J\right)=H_{0}\left(r, p_{r}, J\right)+\varepsilon H_{1}\left(r, p_{r}, J\right) .
$$

When considering the sequence of circular orbits, it is well known 12 that, modulo $O\left(\varepsilon^{2}\right)$, one can replace from the start the radius $r$ on the rhs by its unperturbed circular value as a function of $J, r_{0}^{\text {circ }}(J)$, defined as the solution of $\partial H_{0}\left(r, p_{r}=0, J\right) / \partial r=0$ for a given $J$. (We are assuming here that the $p_{r}$ dependence of $H^{\text {ord }}$ starts at order $p_{r}^{2}$ so that one can consistently set $p_{r}=0$ before considering the $J$ dependence.) This yields for the circular-reduced dependence of $H^{\text {ord }}$ as a function of $J$

$$
H_{\text {circ }}^{\text {ord }}(J)=H_{0}^{\operatorname{circ}}(J)+\varepsilon H_{1}^{\operatorname{circ}}(J)+O\left(\varepsilon^{2}\right),
$$

where $H_{0}^{\operatorname{circ}}(J)=H_{0}\left(r_{0}^{\operatorname{circ}}(J), p_{r}=0, J\right)$ and $H_{1}^{\text {circ }}(J)=$ $H_{1}\left(r_{0}^{\text {circ }}(J), p_{r}=0, J\right)$. Note that the $O(\varepsilon)$ contribution to $H_{\text {circ }}^{\text {ord }}(J)$ is directly equal to the zeroth-order-circular value of the original $O(\varepsilon)$ contribution to the Hamiltonian (4.20) [without any contribution from $\varepsilon r_{1}^{\operatorname{circ}}(J)$, and without any differentiation]. Furthermore, the on-shell vanishing (along exact circular orbits) of $\partial H^{\text {ord }} / \partial r$ also ensures that the circular-reduced $J$ dependence of the orbital frequency $\Omega=\partial H^{\text {ord }}\left(r, p_{r}, J\right) / \partial J$ is simply given by $J$ differentiation of $H_{\text {circ }}^{\text {ord }}(J)$ :

$$
\Omega^{\text {circ }}(J)=\frac{\mathrm{d} H_{\text {circ }}^{\text {ord }}(J)}{\mathrm{d} J}=\Omega_{0}^{\text {circ }}(J)+\varepsilon \frac{\mathrm{d} H_{1}^{\operatorname{circ}}(J)}{\mathrm{d} J}+O\left(\varepsilon^{2}\right),
$$

11 In view, e.g., of the result of Ref. [44] about the consistency of the order reduction of higher-order Hamiltonians, see also Sec. III in Ref. 17] and below.

12 The on-shell vanishing of $\partial H^{\text {ord }} / \partial r$ ensures that when replacing the exact solution $r_{\text {exact }}^{\text {circ }}(J)=r_{0}^{\text {circ }}(J)+\varepsilon r_{1}^{\text {circ }}(J)$, the $\varepsilon r_{1}^{\text {circ }}(J)$ perturbation will only contribute at order $O\left(\varepsilon^{2}\right)$. where $\Omega_{0}^{\text {circ }}(J)=\mathrm{d} H_{0}^{\text {circ }}(J) / \mathrm{d} J$ is its unperturbed value.

From the two results (4.21) and (4.22), it is easy to derive the perturbed value of the function $E^{\Omega}(\Omega) \equiv$ $H_{\text {circ }}^{\text {ord }}\left(J^{\text {circ }}(\Omega)\right)$, where $J^{\text {circ }}(\Omega)$ is the inverse of the function $\Omega^{\text {circ }}(J)$. One gets

$$
E^{\Omega}(\Omega)=E_{0}^{\Omega}(\Omega)+\varepsilon E_{1}^{\Omega}(\Omega)+O\left(\varepsilon^{2}\right),
$$

with

$$
E_{1}^{\Omega}(\Omega)=\left[H_{1}^{\operatorname{circ}}(J)-\frac{\mathrm{d} E_{0}^{\Omega}}{\mathrm{d} \Omega} \frac{\mathrm{d} H_{1}^{\operatorname{circ}}(J)}{\mathrm{d} J}\right]_{J=J_{0}^{\operatorname{circ}}(\Omega)},
$$

where $J_{0}^{\text {circ }}(\Omega)$ is the inverse of the function $\Omega_{0}^{\text {circ }}(J)$.

Let us apply the convenient result (4.24) to the $r_{12}$ scale tail perturbation written as

$$
\varepsilon H_{1}^{\text {tail }\left(r_{12}\right)}=\frac{64}{5} \mu c^{2} \frac{\nu}{j^{10}} \ln \left(\frac{4 \mathrm{e}^{\gamma_{\mathrm{E}}} \Omega r_{0}^{\mathrm{circ}}(J)}{c}\right),
$$

where we leave open for the moment the functional dependence of $\Omega$ within the tail logarithm. In our case $E_{0}^{\Omega}=-\frac{1}{2} \mu c^{2} \widehat{\Omega}^{2 / 3}$ and $j_{0}^{\operatorname{circ}}(\Omega)=\widehat{\Omega}^{-1 / 3}$ (where $\widehat{\Omega} \equiv$ $m \Omega$ ), so that the general result (4.24) yields the simple expression

$$
E_{1}^{\Omega}(\Omega)=\left(1+\frac{1}{3} j \frac{\mathrm{d}}{\mathrm{d} j}\right) H_{1}^{\mathrm{circ}}(j)
$$

which involves a crucial $j$ derivative [by contrast to the derivation of the function $H_{1}^{\text {circ }}(j)=\left[H^{\text {tail }\left(s=r_{12}\right)}\right]^{\operatorname{circ}}(j)$ which involved no differentiation].

Applying Eq. (4.26) to Eq. (4.25) yields

$$
\begin{aligned}
E_{r_{12} \text { tail }}^{\Omega}(\Omega)=- & \frac{448}{15} \mu c^{2} \nu x^{5}\left[\ln \left(\frac{4 \mathrm{e}^{\gamma_{\mathrm{E}}} \Omega r_{0}^{\operatorname{circ}}(j)}{c}\right)\right. \\
& \left.-\frac{1}{7} \frac{\mathrm{d} \ln \Omega}{\mathrm{d} \ln j}-\frac{1}{7} \frac{\mathrm{d} \ln r_{0}^{\operatorname{circ}}(j)}{\mathrm{d} \ln j}\right],
\end{aligned}
$$

where (on shell) $\ln \left(\frac{4 \mathrm{e}^{\gamma_{\mathrm{E}} \Omega r_{0}^{\text {circ }}(j)}}{c}\right)=\ln \left(4 \mathrm{e}^{\gamma_{\mathrm{E}}} x^{\frac{1}{2}}\right)$. As $r_{0}^{\text {circ }}(j)=m j^{2}$, the contribution of the last term 13 in the bracket is $-2 / 7$. Moreover, as our Delaunay-based method led us to have $\widehat{\Omega}=\widehat{\Omega}_{0}^{\text {circ }}(j)=j^{-3}$ in Eq. 44.25) [see notably Eq. (4.11) in Ref. [17]], the penultimate term in the bracket yields the additional term

$$
-\frac{1}{7} \frac{\mathrm{d} \ln \Omega_{0}^{\operatorname{circ}}(j)}{\mathrm{d} \ln j}=+\frac{3}{7}
$$

so that the bracket in Eq. (4.27) becomes

$$
\ln \left(\frac{4 \mathrm{e}^{\gamma_{\mathrm{E}}} \Omega r_{0}}{c}\right)+\frac{3}{7}-\frac{2}{7}=\ln \left(\frac{4 \mathrm{e}^{\gamma_{\mathrm{E}}} \Omega r_{0}}{c}\right)+\frac{1}{7} .
$$

13 Note, in passing, that if we were considering the $s$ scaled tail (with a fixed scale $s$ ) the latter term would be $-\frac{1}{7} \mathrm{~d} \ln s / \mathrm{d} \ln j=0$. 
By contrast, the result claimed in Ref. 21] for that bracket [see Eq. (5.30) there; note that in Eq. (4.27) we factored 448/15, instead of the 224/15 they factor in their Eq. (5.30), to better exhibit the role of $\left.\ln \left(\Omega r_{0}\right)\right]$ reads

$$
\ln \left(\frac{4 \mathrm{e}^{\gamma_{\mathrm{E}}} \Omega r_{0}}{c}\right)-\frac{2}{7} .
$$

The shift $+\frac{3}{7}$, Eq. (4.28), connecting the result (4.30) of Ref. 21] to our [16, 17] result (4.29) is the basis of the claim of Ref. 21] that our value of the constant $C$ should be changed into $C+\frac{3}{7}$ [see Eq. (5.31) in Ref. 21]].

We disagree with this conclusion of Ref. 21] because of the Delaunay-based logic, recalled above, that unescapably leads to the result (4.29) of Refs. [16, 17]. In the next section, we explain why the treatment of Ref. [21] (which, at the effective level, consists in treating $\Omega$ as a constant, instead of a function of $j$ ), though justified within a certain (nonlocal) framework, cannot be correctly combined with the use of the Hamiltonian as conserved energy.

\section{SUBTLETIES IN THE DEFINITION OF THE ENERGY IN THE PRESENCE OF NONLOCAL EFFECTS}

In this section we discuss subtle aspects of the order reduction of nonlocal actions which are automatically incorporated within our approach, but which can easily lead to apparent paradoxes and/or errors when trying to define a conserved energy along circular orbits.

It will be convenient to use an even simpler toy model than the one used above to explain these subtleties, and to further confirm the validity of our result (4.29).

Let us start (as above) with a nonlocal interaction described (in Poincaré-Delaunay variables) by a toy action of the form

$$
S=\int\left[\Lambda \mathrm{d} \lambda+I_{r} \mathrm{~d} \varpi-H^{\text {nonloc }}(t) \mathrm{d} t\right]
$$

with

$$
\begin{aligned}
& H^{\text {nonloc }}(t)=-\frac{1}{2 \Lambda^{2}} \\
&+\varepsilon \int \mathrm{d} \tau \mu(\tau) \Lambda^{\prime n} \Lambda^{n}\left[\cos \left(\lambda^{\prime}-\lambda\right)+O(e)\right]
\end{aligned}
$$

where, as above, $\Lambda \equiv \Lambda(t), \Lambda^{\prime} \equiv \Lambda\left(t^{\prime}\right) \equiv \Lambda(t+\tau)$, etc.

Above, we explained how to completely reduce the nonlocality of this action [i.e. the dependence of $H^{\text {nonloc }}(t)$ on dynamical variables at time $t^{\prime} \neq$ $t$ ] to a purely local dependence. In this reduction the term $\int \mathrm{d} \tau \mu(\tau) \Lambda^{\prime n} \Lambda^{n} \cos \left(\lambda^{\prime}-\lambda\right)$ was replaced by $\int \mathrm{d} \tau \mu(\tau) \Lambda^{2 n} \cos (\Omega(\Lambda) \tau)$ with $\Omega(\Lambda)=1 / \Lambda^{3}$. And we have then seen the crucial role played by the $\Lambda$ dependence of $\Omega$ (corresponding to the $J$ dependence discussed in the previous section).
In order to better see the crucial transition from the original $\cos \left(\lambda\left(t^{\prime}\right)-\lambda(t)\right)$ (where there is no apparent $\Lambda$ dependence) to the final $\cos \left(\Omega(\Lambda)\left(t^{\prime}-t\right)\right)$, we shall study a simple toy model where the original oscillatory factor $\cos \left(\lambda\left(t^{\prime}\right)-\lambda(t)\right) \equiv \cos \left(\frac{\lambda\left(t^{\prime}\right)-\lambda(t)}{t^{\prime}-t}\left(t^{\prime}-t\right)\right)$ is replaced by $\cos \left(\dot{\lambda}\left(t^{\prime}-t\right)\right)$ with $\dot{\lambda} \equiv \mathrm{d} \lambda / \mathrm{d} t$.

A motivation for considering such a simplified dynamics is as follows. If one were formally considering a nonlocality kernel $\mu(\tau)$ which decays sufficiently fast for large $|\tau|$ 's $\left[\right.$ say $\left.\mu(\tau)=\exp \left[-\frac{1}{2}\left(\frac{\tau}{\sigma}\right)^{2}\right] / \sqrt{2 \pi \sigma^{2}}\right]$, one could formally replace in any nonlocal Hamiltonian $H^{\text {nonloc }}=\int \mathrm{d} \tau \mu(\tau) f\left(q, q^{\prime}, p, p^{\prime}\right)$, the time-shifted variables $q^{\prime}=q(t+\tau)$ and $p^{\prime}=p(t+\tau)$ by their Taylor expansions, $q^{\prime}=q+\tau \dot{q}+\frac{1}{2} \tau^{2} \ddot{q}+\cdots$, etc. This then replaces the original nonlocality by a generalized Ostrogradski Hamiltonian depending on the infinite tower of derivatives of the phase-space variables: $H^{\text {ostro }}(q, p ; \dot{q}, \dot{p}, \ddot{q}, \ddot{p}, \cdots)$. The theory of the order reduction of such quasilocal, Ostrogradski-type Hamiltonians has been abundantly treated in the literature, particularly in the context of the PN-expanded dynamics of binary systems (see, e.g., Refs. [41, 42, 44, 45]). By considerations that would be too long to explain here, one can then see that for nonlocalities of the Delaunay type $\int \mathrm{d} \tau \mu(\tau) \Lambda^{\prime n} \Lambda^{n} \cos \left(\lambda^{\prime}-\lambda\right)$, in which one Taylor expands the time-shifted variables, e.g. $\lambda^{\prime}=\lambda(t+\tau)=\lambda+\tau \dot{\lambda}+\frac{1}{2} \tau^{2} \ddot{\lambda}+\cdots, \Lambda^{\prime}=\Lambda(t+\tau)=$ $\Lambda+\tau \dot{\Lambda}+\frac{1}{2} \tau^{2} \ddot{\Lambda}+\cdots$, the crucially delicate nonlocality is only contained in the term $\tau \dot{\lambda}$. The other ones are essentially equivalent to double-zero terms [because of some special structure of the relevant Delaunay-like Hamiltonians, notably a $\mathbb{Z}_{2}$ symmetry transformation combining time reversal $\left(t \rightarrow-t, t^{\prime} \rightarrow-t^{\prime}, \tau \rightarrow-\tau\right)$ and angle reversals $(\lambda \rightarrow-\lambda, \varpi \rightarrow-\varpi)$, and keeping the action variables fixed].

Independently of this motivation, we shall simply use here, for pedagogical purposes, as a simple model of the nonlocal action (5.2), the following first-order Ostrogradski Hamiltonian:

$$
\begin{aligned}
& H_{\text {toy }}^{\text {ostro }}\left(\lambda, \Lambda, \varpi, I_{r} ; \dot{\lambda}, \dot{\varpi}\right)=-\frac{1}{2 \Lambda^{2}} \\
& \quad+\varepsilon \int \mathrm{d} \tau \mu(\tau) \Lambda^{2 n}[\cos (\dot{\lambda} \tau)+O(e)],
\end{aligned}
$$

where the $\dot{\varpi}$ dependence only occurs within the $O(e)$ remainder.

Note that this first-order Ostrogradski Hamiltonian has a quasilocal (but not local) structure in that it depends not only on the phase-space variables $(q, p)$, but also on some of their (first) derivatives (here a dependence on the two $\dot{q}$ 's). Though the "nonlocality" associated with the presence of these derivatives in the Hamiltonian (5.3) might seem trivial, it will actually display the origin of the discrepancy between Ref. 21] and us, and show why the claims of Ref. [21] are unfounded.

In Ref. [44] we have investigated in detail Ostrogradski Hamiltonians depending on $\dot{q}, \dot{p}$ in addition to $q, p$. 
Our main results were the following. First, when considering a general $H^{\text {ostro }}(q, p ; \dot{q}, \dot{p})$ [with action $S_{\text {ostro }}=$ $\left.\int\left(p \dot{q}-H^{\text {ostro }}\right) \mathrm{d} t\right]$, there exists a conserved energy canonically associated with such an Ostrogradski Hamiltonian, which is not given by the Hamiltonian but rather by the following (Noether-theorem-deduced) quantity:

$$
\begin{aligned}
\mathcal{E}^{\text {cons }}(q, p ; \dot{q}, \dot{p})=H^{\text {ostro }} & (q, p ; \dot{q}, \dot{p}) \\
& -\dot{q} \frac{\partial H^{\text {ostro }}}{\partial \dot{q}}-\dot{p} \frac{\partial H^{\text {ostro }}}{\partial \dot{p}} .
\end{aligned}
$$

Second, when considering the case of interest here where

$$
H^{\text {ostro }}(q, p ; \dot{q}, \dot{p})=H_{0}(q, p)+\varepsilon H_{1}(q, p ; \dot{q}, \dot{p}),
$$

one can reduce [modulo $\left.O\left(\varepsilon^{2}\right)\right] H^{\text {ostro }}$ to an ordinary Hamiltonian $H^{\text {ordin }}\left(q^{\prime}, p^{\prime}\right)$ [with action $S^{\text {ordin }}=\int\left(p^{\prime} \mathrm{d} q^{\prime}-\right.$ $\left.H^{\text {ordin }} \mathrm{d} t\right)$ ] by means of the following shifts:

$$
q^{\prime}=q+\frac{\partial H^{\text {ostro }}}{\partial \dot{p}}, \quad p^{\prime}=p-\frac{\partial H^{\text {ostro }}}{\partial \dot{q}} .
$$

In addition the function $H^{\text {ordin }}(q, p)$ is obtained by the naive ("incorrect") order reducing of $H^{\text {ostro }}(q, p ; \dot{q}, \dot{p})$, i.e. by inserting the lower-order equations of motion in $H^{\text {ostro }}$,

$$
H^{\text {ordin }}(q, p)=H^{\text {ostro }}\left(q, p ; \frac{\delta H_{0}}{\delta p},-\frac{\delta H_{0}}{\delta q}\right)+O\left(\varepsilon^{2}\right) .
$$

And, finally, the (Noether) conserved energy (expressed in terms of the original variables) is (numerically) equal to the (order-reduced) ordinary Hamiltonian $H^{\text {ordin }}$ [expressed in terms of the corresponding shifted variables $q^{\prime}$, $p^{\prime}$ of Eq. (5.6)]:

$$
\mathcal{E}^{\mathrm{cons}}(q, p ; \dot{q}, \dot{p})=H^{\mathrm{ordin}}\left(q^{\prime}, p^{\prime}\right)+O\left(\varepsilon^{2}\right) .
$$

Let us now apply the general results (5.4)-(5.8) (recalled from Ref. [4]) to our toy model (5.3). We first note the existence of a (Noetherian) conserved energy, namely

$$
\begin{aligned}
\mathcal{E}_{\text {toy }}^{\text {cons }}(\lambda, \Lambda ; \dot{\lambda}, \dot{\varpi})=H_{\text {toy }}^{\text {ostro }} & (\lambda, \Lambda ; \dot{\lambda}, \dot{\varpi}) \\
& -\dot{\lambda} \frac{\partial H_{\text {toy }}^{\text {ostro }}}{\partial \dot{\lambda}}-\dot{\varpi} \frac{\partial H_{\text {toy }}^{\text {ostro }}}{\partial \dot{\varpi}} .
\end{aligned}
$$

In the circular limit the last term $-\dot{\varpi} \partial H_{\text {tov }}^{\text {ostro }} / \partial \dot{\varpi}$ vanishes because of the vanishing eccentricity 14 . On the other hand the penultimate term does not vanish in the circular limit because it involves a $\tau$-even integrand:

$$
\frac{\partial}{\partial \dot{\lambda}} \int \mathrm{d} \tau \mu(\tau) \cos (\dot{\lambda} \tau)=-\int \mathrm{d} \tau \mu(\tau) \tau \sin (\dot{\lambda} \tau)
$$

14 Here, one should use regular Poincaré variables to rigorously show that the $O(e)$ terms in 5.3 do not contribute when $e \rightarrow 0$. so that

$$
\begin{aligned}
& \mathcal{E}_{\text {circ }}^{\text {cons }}(\Lambda, \dot{\lambda})=-\frac{1}{2 \Lambda^{2}} \\
& \quad+\varepsilon \Lambda^{2 n} \int \mathrm{d} \tau \mu(\tau)[\cos (\dot{\lambda} \tau)+\dot{\lambda} \tau \sin (\dot{\lambda} \tau)]
\end{aligned}
$$

In the circular limit the extra term $-\dot{\lambda} \partial H / \partial \dot{\lambda}$ is independent of time and cannot therefore be guessed simply by looking at the equations of motion.

In other words, our toy model shows that, when working with a non-order-reduced, nonlocal Hamiltonian, the Noetherian conserved energy differs from the Hamiltonian, even in the circular limit. This is another way to view (part of) the discrepancy with the derivation of the function $E(\Omega)$ in Ref. [21]. Indeed, the latter reference works within a (non-order-reduced) nonlocal framework, but uses as conserved circular energy the circularreduced Hamiltonian itself, i.e., in our toy model, simply $H_{\text {toy }}^{\text {ostro }}(\Lambda, \dot{\lambda})$, which differs from the "good" (Noetherian) conserved energy (5.9) and (5.11).

Another useful outcome of the results (5.4)-(5.8) recalled above is that, while there is no need to shift the angular variables $\lambda, \varpi$ (because $\delta \lambda=\partial H^{\text {ostro }} / \partial \dot{\Lambda}=0$, $\left.\delta \varpi=\partial H^{\text {ostro }} / \partial \dot{I}_{r}=0\right)$, one must shift the action variables if one wishes to have an order-reduced dynamics. The first fact says in particular that, in the circular limit, the orbital angular frequency $\dot{\lambda}=\dot{\lambda}^{\prime}$ is unambiguous and is given either by the (functional) derivative

$$
\begin{aligned}
\dot{\lambda} & =\frac{\delta H_{\text {toy }}^{\text {ostro }}(\Lambda, \dot{\lambda})}{\delta \Lambda} \\
& =\frac{1}{\Lambda^{3}}+2 n \varepsilon \Lambda^{2 n-1} \int \mathrm{d} \tau \mu(\tau) \cos (\dot{\lambda} \tau),
\end{aligned}
$$

in which the frequency $\dot{\lambda}$ in the argument of the cosine is treated as a constant, or [up to $O\left(\varepsilon^{2}\right)$ corrections] by the (ordinary) derivative of the order-reduced, ordinary Hamiltonian

$$
\begin{aligned}
H_{\text {toy }}^{\text {ordin }} & \left(\lambda^{\prime}, \varpi^{\prime}, \Lambda^{\prime}, I_{r}^{\prime}\right)=-\frac{1}{2 \Lambda^{\prime 2}} \\
& +\varepsilon \Lambda^{\prime 2 n} \int \mathrm{d} \tau \mu(\tau)\left[\cos \left(\frac{\tau}{\Lambda^{\prime 3}}\right)+O(e)\right],
\end{aligned}
$$

which yields (in the circular limit) an orbital frequency given by

$$
\begin{aligned}
\dot{\lambda}^{\prime} & =\frac{\partial H_{\text {toy }}^{\text {ordin }}}{\partial \Lambda^{\prime}} \\
& =\frac{1}{\Lambda^{\prime 3}}+\varepsilon \frac{\partial}{\partial \Lambda^{\prime}}\left[\Lambda^{\prime 2 n} \int \mathrm{d} \tau \mu(\tau) \cos \left(\frac{\tau}{\Lambda^{\prime 3}}\right)\right] .
\end{aligned}
$$

Contrary to Eq. (5.12) the $\Lambda^{\prime}$ derivative on the rhs of Eq. (5.14) now acts both on the prefactor $\Lambda^{\prime 2 n}$ and on the argument of the cosine. Note that in the real $4 \mathrm{PN}$ problem the power $2 n$ is equal to -10 and the weight 
function $\mu(\tau)$ is either $\operatorname{Pf}_{2 s / c}|\tau|^{-1}$ or $\operatorname{Pf}_{2 r_{12} / c}|\tau|^{-1}$ (as one chooses). Using, say, the latter $\left(r_{12^{-}}\right)$choice, and, e.g., Eq. (5.8) of Ref. [16], one has then

$$
\varepsilon \Lambda^{-10} \int_{-\infty}^{+\infty} \mathrm{d} \tau \mu(\tau) \cos (\dot{\lambda} \tau)=-\varepsilon \Lambda^{-10} \ln \left(\frac{4 \mathrm{e}^{\gamma_{\mathrm{E}}} \dot{\lambda} r_{12}}{c}\right)
$$

The result (5.15) displays the structure of the tail contribution to the Hamiltonian, as in Eq. (4.25). However, we now see that there are two different ways of dealing with this contribution. (The consistency of these two different ways will be discussed below.) The difference between the two calculations (5.12) and (5.14) parallels what we explained above about the difference between the prescription used by Ref. 21] (treating $\dot{\lambda}$ as a constant within the argument of the tail logarithm) and the prescription used by us [16, 17] (where $\Omega$ was a function of $j$ ). Indeed, there are two different methods. The first method is to stay within a non-order-reduced, nonlocal framework (as is the case of Ref. [21]), and then consider the orbital frequency $\dot{\lambda}$ as being a priori unrelated to $j$ [so that the additional term (4.28) is a priori absent as in the calculation of Ref. [21]], but, one must then take as conserved energy the quantity (5.9), which differs from the Hamiltonian in the circular limit (contrary to what was used in Ref. [21]). The second method is to work within an order-reduced, local Hamiltonian (as is the case of Ref. [17]), in which case one must take into account the additional term (4.28), and one can (and must) use as conserved energy the (order-reduced, ordinary) Hamiltonian when computing the function $E(\Omega)$ (as was done in Refs. [16, 17]).

The detailed reasoning we just made shows that the calculation (5.14) [involving the ordinary, order-reduced Hamiltonian (5.13)] is not only self-consistent (as we already argued above) but, most importantly, is consistent with the nonlocal dynamics of the original Ostrogradski Hamiltonian (5.3), under the following two conditions: (i) the conserved energy associated with the nonlocal description (5.3) should not be equated (contrary to the prescription used in Ref. [21]), when considering the circular limit, with the value of $H^{\text {ostro }}$, but rather with Eqs. (5.9) and (5.11), and (ii) the variables $\lambda, \Lambda, \varpi, I_{r}$ entering the (weakly) nonlocal dynamics (5.3) cannot be equated with the variables $\lambda^{\prime}, \Lambda^{\prime}, \varpi^{\prime}, I_{r}^{\prime}$ entering the corresponding order-reduced ordinary dynamics (5.13). More precisely, the link between the two sets of variables is $\lambda^{\prime}=\lambda$, $\varpi^{\prime}=\varpi$ but

$$
\begin{aligned}
& \Lambda^{\prime}=\Lambda-\frac{\partial H_{\text {toy }}^{\text {ostro }}}{\partial \dot{\lambda}}=\Lambda+\varepsilon \Lambda^{\prime 2 n} \int \mathrm{d} \tau \mu(\tau) \tau \sin (\dot{\lambda} \tau), \\
& I_{r}^{\prime}=I_{r}-\frac{\partial H_{\text {toy }}^{\text {ostro }}}{\partial \dot{\varpi}}=I_{r}+O(e) .
\end{aligned}
$$

It is easily checked that the shift (5.16a) ensures the consistency between the two (different) functions $\Lambda \rightarrow \dot{\lambda}$,
Eq. (5.12), and $\Lambda^{\prime} \rightarrow \dot{\lambda}^{\prime}=\dot{\lambda}$, Eq. (5.14). It is moreover checked that the conserved energy $\mathcal{E}_{\text {circ }}^{\text {cons }}(\Lambda, \dot{\lambda})$, Eq. (5.11), becomes numerically equal to the ordinary Hamiltonian energy (5.13) when also taking into account the shift (5.16a).

Summarizing so far: we have explicitly shown, by two different approaches (one of them being the one used in Refs. [16, 17], and the other being as close as possible to the one used in Ref. [21]), that the correct $\left(r_{12}\right.$-scaled) tail contribution to the (Noetherian) conserved energy (along circular orbits) expressed in terms of the orbital frequency is given by Eqs. (4.27) and (4.29), in agreement with our results [16, 17].

\section{INCOMPATIBILITY BETWEEN THE $C$-MODIFIED FOKKER-ACTION RESULT OF REF. 21] AND SELF-FORCE RESULTS}

An immediate consequence of the result of the previous section is that the determination in Ref. [21] of the ratio between the IR cutoff scale $r_{0}$ and the scale $s_{0}=r_{0}^{\prime} \mathrm{e}^{-11 / 12}$ entering the computation of the tailtransported near-zone metric perturbation [18] is incorrect. In other words, in terms of the notation of Ref. [16] [and of Eqs. (2.3)-(2.4) above] one should add to the Hamiltonian of Ref. [21] an extra term $\Delta C F\left[\mathbf{x}_{a}, \mathbf{p}_{a}\right]$ with

$$
\Delta C=-\frac{3}{7}
$$

If we understand correctly the notation used in Ref. 21], their (ambiguity) parameter $\alpha$ [in their Eq. (3.9)] is equivalent to our (ambiguity) parameter $-C$ modulo a fixed, additive constant linked to the difference between the two schemes. It would then mean that the correct value of their $\alpha$ should be

$$
\alpha_{\mathrm{B}^{3} \mathrm{FM}}^{\text {new }}=\alpha_{\mathrm{B}^{3} \mathrm{FM}}+\frac{3}{7}=\frac{811}{672}+\frac{3}{7}=\frac{157}{96},
$$

instead of the value, here denoted $\alpha_{\mathrm{B}^{3} \mathrm{FM}}$, in their Eq. (4.30). (Note that the denominator of $\alpha_{\mathrm{B}^{3} \mathrm{FM}}^{\text {new }}$ is simpler than that of $\alpha_{\mathrm{B}^{3} \mathrm{FM}}$ : namely $96=2^{5} \times 3$ while $672=7 \times 96=2^{5} \times 3 \times 7$.)

Modifying the Hamiltonian of Ref. [21] by $+\Delta C F$ then modifies the difference $H_{\mathrm{B}^{3} \mathrm{FM}}^{\text {new }}-H_{\mathrm{DJS}}$ into an expression of the same form as the rhs of Eq. (3.3) but with the following new values of the three coefficients $(a, b, c)$ :

$$
(a, b, c)_{\mathrm{B}^{3} \mathrm{FM}}^{\mathrm{new}}=(a, b, c)_{\mathrm{B}^{3} \mathrm{FM}}+\Delta C \frac{16}{15}(-11,12,0) .
$$

Inserting $\Delta C=-\frac{3}{7}$ from Eq. (6.1), and using Eq. (3.4) above, yields

$$
(a, b, c)_{\mathrm{B}^{3} \mathrm{FM}}^{\text {new }}=\frac{1}{315}(3013,-902,902) .
$$

The latter result is equivalent [modulo a gauge transformation, Eq. (3.6), with $g=288 / 315]$ to the rhs of Eq. 
(5.34b) in Ref. 21], but with the different meaning that it is the Hamiltonian of Ref. 21] on the lhs which has to be shifted instead of $H_{\text {DJS }}$.

Inserting Eq. (6.4) into our general formulas (3.5a)(3.5b) then leads to the following additional contributions (compared to our results [17]) to the EOB potentials $A$ and $\bar{D}$ entailed by the above modified version of the Hamiltonian of Ref. 21]:

$$
\begin{aligned}
\delta^{\text {new }} A & =0, \\
\delta^{\text {new }} \bar{D} & =-\frac{34}{9} \nu u^{4} .
\end{aligned}
$$

The first result, Eq. 6.5), shows that our prescription (6.1) for modifying the result of Ref. 21] now yields a value of the EOB $A$ potential at $4 \mathrm{PN}$ in agreement with our result [16, 17] (and with the many existing SF computations of $A$ recalled above). On the other hand, we see in Eq. (6.6) that, even after our correction, the $4 \mathrm{PN}$ contribution to the EOB $\bar{D}$ potential predicted by the Fokker-action, harmonic-gauge computation of Ref. [21] significantly differs from our result [17.

In terms of the gauge-invariant $4 \mathrm{PN}$-level parameters $a_{5}^{c}, \bar{d}_{4}^{c}, \rho_{4}^{c}$ introduced above, the results (3.9), (3.10), and (3.12) above are now replaced by

$$
\begin{aligned}
& \delta^{\mathrm{B}^{3} \mathrm{FM}^{\prime}} a_{5}^{c}=0, \\
& \delta^{\mathrm{B}^{3} \mathrm{FM}^{\prime}} \bar{d}_{4}^{c}=-\frac{34}{9}=-3.77777 \cdots, \\
& \delta^{\mathrm{B}^{3} \mathrm{FM}^{\prime}} \rho_{4}^{c}=-\frac{34}{9}=-3.77777 \cdots
\end{aligned}
$$

As we already said above, there are many (analytical and numerical) SF results showing that the $4 \mathrm{PN}$ coefficients $\bar{d}_{4}^{c}$ or $\rho_{4}^{c}$ nicely agree with the ADM result [17]. (Let us, in particular, recall the recent analytical confirmations [32, 46].) Numerical tests making use of the eccentric first law 28] yield a limit $\left|\delta \bar{d}_{4}^{c}\right|^{\text {num }}<0.05$ on any possible deviation $\delta \bar{d}_{4}^{c}$ away from our analytical result (see discussion and references, in Ref. [46] and above). As for direct dynamical limits on the $4 \mathrm{PN}$-level coefficient of the precession function $\rho(u)$ we have displayed them in Eqs. (2.13) and (2.14) above. While the old limit (2.13) 34] would be compatible with the modified result (6.9), the recent limit (2.14) [35] is in violent disagreement with Eq. (6.9).

\section{SUGGESTION FOR ADDING MORE IR AMBIGUITY PARAMETERS IN REF. [21]}

In view of the strong discrepancy (6.8) and (6.9), remaining after making use of the sole IR ambiguity parameter $C=-\alpha+$ const, we suspect that the harmonic Fokker action derived in Ref. 21 has to be modified not only by the shift (6.1) of $C$ (or $-\alpha$ ), but by suitable shifts of further ambiguity parameters entering their present computation.
An independent argument suggesting the presence of more ambiguities in their calculations than the mere $C F[\mathbf{r}, \mathbf{p}]$ ambiguity (which is also present in our ADM calculation, and was only fixed by using the analytical result of Ref. [13]) is presented next.

On the one hand, though the ADM calculation is not manifestly Poincaré invariant, and involves both UV and IR divergences that we had to regularize, our final result is Poincaré invariant as is, without having to invoke any correcting contact transformation. In particular, the 4PN ADM Hamiltonian is manifestly invariant under spatial translations, i.e. only depends on the relative position $\mathbf{y}_{12}=\mathbf{y}_{1}-\mathbf{y}_{2}$ of the two particles.

On the other hand, the core of the harmoniccoordinates calculation of Ref. 21] is the evaluation of the (local) Fokker Lagrangian [their Eqs. (2.20) and (4.20)]

$$
L_{g}=\mathrm{FP} \int \mathrm{d}^{3} x\left(\frac{r}{r_{0}}\right)^{B} \mathcal{L}_{g},
$$

where

$$
\mathcal{L}_{g} \sim c^{4}[\bar{h} \square \bar{h}+\bar{h} \partial \bar{h} \partial \bar{h}+\cdots+\bar{h} \bar{h} \bar{h} \bar{h} \partial \bar{h} \partial \bar{h}],
$$

in which one inserts the formal, near-zone PN expansion of the metric variables, computed in harmonic coordinates. Both the harmonic Fokker Lagrangian density $\mathcal{L}_{g}$ and the (time-symmetric) harmonic metric are formally Poincaré covariant. The Lagrangian $L_{g}$ should then vary, under Poincaré transformations, as the time component of a four-vector, except if the (non-Poincaréinvariant) IR regulator $\mathrm{FP}\left(|\mathbf{x}| / r_{0}\right)^{B}$ introduces violations of Poincaré invariance. Note, in particular, that all the ingredients of the calculation are manifestly invariant under spatial translations, except for the IR regulator. However, Bernard et al. 21] stated that the raw result of the computation (7.2) is not translation invariant, but depends on the individual positions $\mathbf{y}_{A}$ of the particles. They found that the offending terms can be removed by some shifts $\xi_{A}$ of the positions $\mathbf{y}_{A}$ so as to yield a manifestly Poincaré covariant (modulo a time derivative) Lagrangian, say $L_{g}^{\text {shifted }}$. An inspection of the needed shifts (in their Appendix C) reveals a very large number of offending non-translation-invariant terms which are mostly connected with IR divergences $\left[\propto \ln \left(r_{12} / r_{0}\right)\right]$. Moreover, there are terms in the shifts $\xi_{A}$ which combine both IR and UV divergences $\left[\propto \ln \left(r_{12} / r_{1}^{\prime}\right)\right]$. By contrast with the ADM calculation which, in spite of its nonmanifest Poincaré invariance, ends up with a Poincaréinvariant (and, in particular, translation-invariant) regularized dynamics, we consider that several features of the harmonic-coordinate calculation strongly suggest the presence of more ambiguities in the final result for $L_{g}$ than the $C F[\mathbf{r}, \mathbf{p}]$ one, namely: 1) the large zoology of nontranslationally invariant IR-divergent terms; 2) the mixing of IR and UV divergences, together with the use of a method which uses Hadamard regularization as an intermediate step for UV divergences (before "correcting" it by extra dimensional-regularization terms); and, 
3) the known fact that harmonic coordinates imply a worse behavior in the outer near zone than ADM coordinates [notably because the ten metric components $g_{\mu \nu}$ propagate with the velocity of light in harmonic coordinates, while, in ADM coordinates, only $h_{i j}^{\mathrm{TT}}=O\left(1 / c^{4}\right)$ does so].

We therefore suggest that Bernard et al. 21] should (similarly to what happened at the $3 \mathrm{PN}$ level when using only Hadamard-type regularization [44, 47 49]) acknowledge the presence of further ambiguities in their result, parametrized by new ambiguity parameters. We shall assume here that these ambiguities affect the three discrepant terms (3.3) and only them.

In view of the unavoidable presence of the $C F[\mathbf{r}, \mathbf{p}]$ ambiguity, and of the possibility of making the gauge transformation (3.6), the minimal number of extra ambiguity parameters that need to be introduced is one. For instance, we could introduce a single ambiguity parametrized by adding to the result of Ref. 21] a term in the Hamiltonian of the form

$$
\delta^{\Delta a} H=+\Delta a \frac{G^{4} M m_{1}^{2} m_{2}^{2}}{c^{8} r_{12}^{4}}\left(\frac{\left(\mathbf{n}_{12} \cdot \mathbf{p}_{1}\right)}{m_{1}}-\frac{\left(\mathbf{n}_{12} \cdot \mathbf{p}_{2}\right)}{m_{2}}\right)^{2},
$$

which has the effect of changing $(a, b, c)$ into $(a+\Delta a, b, c)$. Such a change does not affect the EOB $A$ potential [which is $\propto 2(b+c)$, Eq. (3.5a) ], but affects the EOB $\bar{D}$ potential [which is $\propto 2(a+4 b)$, Eq. 3.5b)]. We conclude that one must add to their result in addition to $\triangle C F$, with $\Delta C=-3 / 7$, Eq. (3.3), the extra contribution (7.3) with

$$
\Delta a=+\frac{17}{9} .
$$

It is also possible that part of the ambiguities in their calculation are parametrized by a transformation of the type (3.6), but such a transformation has no gauge-invariant impact on the dynamics as it is induced by a canonical transformation with generating function $\propto \nu \mathbf{n}_{12} \cdot\left(\mathbf{p}_{1} / m_{1}-\mathbf{p}_{2} / m_{2}\right) r_{12}^{-3}$ (corresponding to changes of $\mathbf{r}_{1}$ and $\mathbf{r}_{2}$ in a purely radial direction). (Note, however, that even such "gauge ambiguities" will affect the computation of the radiative quadrupole moment of the system, see below.)

\section{CONCLUSIONS}

Let us summarize our main conclusions concerning the conservative dynamics of a binary system at the $4 \mathrm{PN}$ approximation.

- We have reviewed the numerous confirmations of the correctness of the 4PN ADM dynamics [14, 16, 17]. When decomposed in powers of the symmetric mass ratio $\nu$, all its elements have been confirmed, piecewise, by independent computations. In particular, the crucial linear-in- $\nu$ terms (at the first-selfforce level) have been confirmed, both analytically and numerically, by several self-force results.
- By contrast we have computed the periastron precession induced by the recently reported harmonic Fokker-action 4PN dynamics [21] and found it to be in violent contradiction with existent direct numerical self-force computations [35]: compare Eq. (3.12) to Eq. 2.14.

- After reviewing the logical basis and the consistency of the ADM-Delaunay-EOB derivation of Refs. [16, 17], we pointed out two different flaws in the Fokker-action harmonic-coordinates computation of Ref. 21]: (i) their computation of the functional link, along circular orbits, between the energy and the orbital frequency involves (when viewed within the approach of Sec. V) a correct treatment of nonlocal (tail) effects but a flawed assumption about the value of the physically relevant conserved energy; (ii) after correcting for the previous issue, there remains a violent incompatibility between their theoretically predicted periastron precession and recent dynamical self-force computations [compare Eq. (6.9) to Eq. (2.14)].

- We suggest that the result of Ref. [21] must be corrected by adding (at least) two infrared ambiguity parameters to the Hamiltonian form of their result: 1) $\Delta C=-\Delta \alpha=-\frac{3}{7}$, Eq. 6.1); and 2) $\Delta a=+\frac{17}{9}$, Eqs. (7.3) and (7.4) [modulo a gauge transformation, Eq. (3.6)]. Let us again emphasize that our suggested corrections represent only two rather minor adjustments among hundreds of terms that agree between two very difficult independent calculations, which have used different methods and different gauges.

- Before attempting a $4 \mathrm{PN}-$ level computation of gravitational-wave emission (in the more convenient harmonic coordinates), it will be necessary to confirm, within the framework of Ref. 21], the presence, and value, of the couple of ambiguities (notably $\Delta a$ and $g$ ) mentioned above.

\section{ACKNOWLEDGMENTS}

T.D. thanks Leor Barack and Maarten van de Meent for informative email exchanges. The work of P.J. was supported in part by the Polish NCN Grant No. UMO2014/14/M/ST9/00707.

\section{Appendix A: Fokker-type actions and tail Hamiltonian in the ADM formalism}

Before discussing several technical aspects of the (4PNlevel) tail contribution to the ADM dynamics, let us start by recalling some general features, common to the ADM action, and the harmonic-Fokker one. 
Both actions are Fokker-type actions, obtained (as was done for the electromagnetic interaction in Ref. [50]) by eliminating (or "integrating out") the field, say $\phi$, in the total action, say $S_{\text {tot }}\left[\phi, x_{a}\right]$, so as to obtain a reduced action $S_{\text {red }}\left[x_{a}\right]$ for the conservative dynamics of the particle worldlines $x_{a}^{\mu}\left(s_{a}\right)$. At the lowest order, where one considers the linear coupling of the field to the worldlinedistributed source $J\left[x_{a}\right]$, the elimination of the field is obtained by a (formal) integration by parts leading to a replacement of the type (we omit indices, arguments and integration measures)

$$
\int\left[-\frac{1}{2}(\partial \phi)^{2}+\phi J\right]=\int \frac{1}{2} J G J,
$$

where $G$ denotes the appropriate time-symmetric (halfretarded-half-advanced) Green's function, which solves $\square \phi=-J$ as $\phi=G J$. When considering, higher-order (nonlinear) couplings, one uses an iterative solution of the field equations, and the elimination of the field then leads to a Feynman-like expression for the reduced action involving the concatenation of sources, propagators and vertices, say

$$
S_{\text {red }}\left[x_{a}\right]=\int \frac{1}{2} J G J+\int \mathcal{V}_{3}(G J, G J, G J)+\cdots,
$$

where $\mathcal{V}_{3}$ denotes a cubic vertex. See, e.g., Ref. [51] which discussed in detail the corresponding diagrammatic representation of the reduced harmonic-gauge action. See also Fig. 1 in Ref. 1], and the discussion below Eq. (2.9) there, for a diagrammatic representation of the reduced $\mathrm{ADM}$ action.

Let us note in passing that we disagree on some formal aspects of the definition of a Fokker action in Ref. 21]. Indeed, contrary to the $4 \mathrm{PN}$ ADM computation which explicitly uses the time-symmetric propagation of the TT degrees of freedom (see Sec. IV of Ref. [14]), Ref. [21] described their calculation (at least for the quadrupolar tail contribution, but possibly not for the main contributions to the local action) as if they formally worked with the nonconservative dynamics of a binary system interacting via the retarded Green's function. They then argue that the terms associated with radiation damping only contribute time-derivative terms to the action they computed. We understand technically why this works out formally; however, we object to such a conceptual framework, because the use of a retarded Green's function physically implies a nonconservative dynamics. Indeed, let us recall that, before formally eliminating the field $\phi$, the variation of the original action $S_{\text {tot }}\left[\phi, x_{a}\right]$ with respect to the particle worldlines $x_{a}^{\mu}$ yields (in general relativity) the equations of motion of the particles (as geodesics in the metric $g_{\mu \nu}$ ). This shows that when solving the field $\phi \sim g_{\mu \nu}$ by means of a retarded Green's function, one necessarily gets a dynamics involving, retarded (and therefore dissipative [52]) interactions. It is therefore conceptually incorrect to compute an action supposed to describe a conservative dynamics by using a retarded Green's function. (From the technical point of view, the inconsistency of using a retarded field when formally integrating by parts $S_{\mathrm{tot}}\left[\phi, x_{a}\right]$, while replacing $\phi$ as a functional of the worldlines, shows up in nonvanishing surface terms.) We therefore define the conservative dynamics of gravitationally interacting particles by integrating out the field obtained by iteratively solving the field equations by time-symmetric propagators. It is not clear to us that, when working as we do here with nonlinear aspects of general relativity, there is an alternative route for defining a conservative dynamics. [The additional, nonlocal, time-antisymmetric radiationreaction force needed to transform, at the $4 \mathrm{PN}$ level, the conservative dynamics into the physically more directly meaningful dissipative (causal) dynamics entailed by retarded interactions was exhibited in Sec. VI of Ref. [16].]

Another point we want to make here is that, when working with such reduced actions, one can consider that the dynamics is defined by the final worldline action (A2), and one does not need to worry about the convergence properties of the initial field contributions to $S_{\text {tot }}\left[\phi, x_{a}\right]$ [such as $-\frac{1}{2} \int(\partial \phi)^{2}$, which are no longer relevant after the elimination of the field $\phi$. For instance, in the trivial case of the linear coupling (A1), the transition between the initial total action $S_{\text {tot }}\left[\phi, x_{a}\right]$, and the reduced action $\int \frac{1}{2} J G J$ is well defined 15 for unbound (hyperbolictype) motions. On the other hand, if one considers instead bound (elliptic-like) motions, the spatial integral of the field energy (when considering the Hamiltonian) associated with $\frac{1}{2}(\partial \phi)^{2}$ is linearly IR divergent for bound (quasiperiodic) motions, because both $\phi$ and its partial derivatives only decay as $O(1 / r)$ at spatial infinity, when considering, as must be done in a Fokker-action calculation, the time-symmetric solution $\phi_{\text {sym }}[$ which is a superposition of standing waves of the rough type $\cos (\omega r) / r]$. (Related IR divergences have been recently pointed out in Ref. [53], within the context of second-order gravitational self-force theory.) But, we can consider that the dynamics of bound motions is defined by taking the analytic continuation (from the unbound case to the bound one) of the (a priori better IR-behaved) reduced action $S_{\text {red }}\left[x_{a}\right]$, Eq. A2 . [For instance, the linear interaction $\int \frac{1}{2} J G J$ features no IR divergences linked to the slow spatial decay of the (eliminated) field $\phi$.] We adopt here this attitude (for the reduced ADM action). This is why we wished to point out in Sec. IV that the oscillatory nature of the integrand $I_{i j}^{\prime(3)} I_{i j}^{(3)}$ in the nonlocal piece of the $4 \mathrm{PN}$ action ensured the convergence of the integral over the relative time $\tau=t^{\prime}-t$. We leave to future work a more detailed analysis of the convergence properties of the ADM-Fokker action.

Reference [16] showed the necessity of including in the ADM action a tail-related contribution

15 After factoring the usual infinity $-\int \mathrm{d} t E_{\text {tot }}$ associated with the dynamics of the center of mass of the system. 
$-\int \mathrm{d} t H^{\text {tail sym }(s)}$, with

$$
\begin{aligned}
H_{4 \mathrm{PN}}^{\mathrm{tail} \mathrm{sym}(s)}(t)= & -\frac{1}{5} \frac{G^{2} M}{c^{8}} I_{i j}^{(3)}(t) \\
& \times \operatorname{Pf}_{2 s / c} \int_{-\infty}^{+\infty} \frac{\mathrm{d} v}{|v|} I_{i j}^{(3)}(t+v),
\end{aligned}
$$

by recalling the existence (first shown by Blanchet and Damour [18]) of a fundamental breakdown of the standard PN scheme at the $4 \mathrm{PN}$ level. Indeed, when computing conservative effects, as is appropriate both in the computation of the reduced ADM action, and in that of the harmonic action, the standard PN scheme is based on a formal near-zone expansion of the time-symmetric gravitational propagator, say

$$
\begin{aligned}
\square_{\mathrm{sym}}^{-1} & =\left(\Delta-\frac{1}{c^{2}} \partial_{t}^{2}\right)^{-1} \\
& =\left(\Delta^{-1}+\frac{1}{c^{2}} \Delta^{-2} \partial_{t}^{2}+\cdots\right) \delta\left(t-t^{\prime}\right) .
\end{aligned}
$$

At the $4 \mathrm{PN}$ level the near-zone solution generated by using the PN expansion A4 is incomplete (as a nearzone solution), and must be completed by adding to it a $4 \mathrm{PN}$-level homogeneous solution of the (linearized) field equations (regular within the source) which is a nonlocalin-time functional of the dynamical configuration of the system (related to tail effects).

Reference [18] showed that in a suitable coordinate gauge the additional, $4 \mathrm{PN}$-level, tail-related homogeneous metric perturbation $h_{\mu \nu 4 \mathrm{PN}}^{\text {nonloc }}$ contains only a quadrupolar time-time component of the form

$$
h_{004 \mathrm{PN}}^{\text {nonloc }}=\frac{1}{2} x^{i} x^{j} H_{i j}^{\text {nonloc }}(t),
$$

with the nonlocal function of time $H_{i j}^{\text {nonloc }}(t)$ given by

$$
H_{i j}^{\text {nonloc }}(t)=-\frac{8}{5} \frac{G^{2} M}{c^{10}} \operatorname{Pf}_{2 s / c} \int_{-\infty}^{+\infty} \frac{\mathrm{d} \tau}{|\tau|} I_{i j}^{(6)}(t+\tau) .
$$

The pure, quasi-Newtonian, nature of the additional (nonlocal) metric perturbation (A5) allowed us, in Ref. [16], by considering the corresponding additional term in the equations of motion of the system, to (i) derive the corresponding additional (time-symmetric) nonlocal contribution (A3) to the action (modulo a time derivative) and, (ii) to check the consistency with the purely retarded nature of the nonlocality in the equations of motion when adding the (time-antisymmetric) radiation-reaction force $\mathcal{F}_{4 \mathrm{PN}}^{\mathrm{rad} \text { reac }}$

Reference 21] formally showed how to recover the nonlocal contribution (A3) from the harmonic-gauge Fokker action, without considering the equations of motion, and without using the simplifying features of the special gauge leading to Eq. A5 only. We wish here both to show how the same result can be achieved within the ADM formalism and why the procedure advocated in
Ref. 21] is, in our opinion, exceedingly formal and actually ill-defined because of its sensitivity to nonconvergent integrations by parts.

In the ADM formalism, before eliminating the TT variables, the Routhian 16 [47] reads (in the following we often use without warning the ADM convention $16 \pi G=1$ )

$$
\begin{aligned}
R\left[\mathbf{x}_{a}, \mathbf{p}_{a}, h_{i j}^{\mathrm{TT}}, \dot{h}_{i j}^{\mathrm{TT}}\right] & =\int \mathrm{d}^{3} x\left[\frac{1}{4}\left(\partial_{k} h_{i j}^{\mathrm{TT}}\right)^{2}\right. \\
& \left.-\frac{1}{4 c^{2}}\left(\dot{h}_{i j}^{\mathrm{TT}}\right)^{2}-\frac{1}{4} h_{i j}^{\mathrm{TT}} J_{i j}+\cdots\right],
\end{aligned}
$$

where the leading-order source term for $h_{i j}^{\mathrm{TT}}$ reads

$$
J_{i j}=2 \sum_{a} \frac{p_{a i} p_{a j}}{m_{a}} \delta\left(\mathbf{x}-\mathbf{x}_{a}\right)+\frac{1}{2} \partial_{i} \phi \partial_{j} \phi+\cdots,
$$

and where the ellipsis denotes higher-order terms that we shall not explicitly need here. Note that the longitudinal field degrees of freedom, $\phi$ and $\tilde{\pi}^{i j}$, are viewed as being expressed in terms of the other degrees of free$\operatorname{dom}\left(\mathbf{x}_{a}, \mathbf{p}_{a}, h_{i j}^{\mathrm{TT}}, \dot{h}_{i j}^{\mathrm{TT}}\right)$ by using the constraints (which are elliptic in nature).

The key point is that the ADM analog of the decomposition recalled above, $g_{\mu \nu}^{\mathrm{sym}}=g_{\mu \nu}^{\text {loc PN }}+h_{\mu \nu}^{\text {nonloc }}$, reads

$$
h_{i j}^{\mathrm{TT} \text { sym }}=h_{i j}^{\mathrm{TT} \text { loc PN }}+h_{i j}^{\mathrm{TT} \text { nonloc }} .
$$

Here, $h_{i j}^{\mathrm{TT}}$ loc PN is the usual, near-zone, time-symmetric $\mathrm{PN}$-expanded formally local solution of the field equation for $h_{i j}^{\mathrm{TT}}$, namely

$$
\square h_{i j}^{\mathrm{TT}}=-\frac{1}{2} \delta_{i j}^{\mathrm{TT} k l} J_{k l},
$$

as taken into account in the usual ADM version of the PN expansion [14, 47]. On the other hand, the additional, tail-transported term $h_{i j}^{\mathrm{TT}}$ nonloc is, again, when viewed in the near zone, a homogeneous solution of the (linearized) field equations for $h_{i j}^{\mathrm{TT}}$. The latter nonlocal, tail-transported homogeneous solution is directly deducible from the results of Schäfer [54] who studied radiation and tail effects, within the ADM framework, both in the wave zone and in the near zone. The Green's functions in the latter paper [see Eq. (22) there] yield the following tail-modified radiation-reaction contribution to the (usual, retarded) solution:

$$
\begin{aligned}
h_{i j}^{\mathrm{TT} \text { reac }}(t) & =-\frac{4 G}{5 c^{5}}\left[\mathcal{J}_{i j}^{(1)}(t)\right. \\
+ & \left.\frac{4 G M}{c^{3}} \int_{0}^{\infty} \mathrm{d} \tau \ln \left(\frac{c \tau}{2 s}\right) \mathcal{J}_{i j}^{(3)}(t-\tau)\right],
\end{aligned}
$$

16 The Routh functional is a Hamilton function for the bodies but a Lagrange functional for the field degrees of freedom. 
where

$$
\mathcal{J}_{i j}(t)=\int \mathrm{d}^{3} x J_{\langle i j\rangle}(t, \mathbf{x})
$$

is the trace-free part of the spatial integral of the source (A8) of the field equation (A10) for $h_{i j}^{\mathrm{TT}}$. Note that the tail-modified reactive contribution (A11) to $h_{i j}^{\mathrm{TT}}$ is simply a function of time. This is the anti-Newtonian version of the gauge (A5), namely $h_{i j}=F_{i j}(t)$ instead of $h_{00}=$ $\frac{1}{2} F_{i j}^{(2)} x^{i} x^{j}$, with the same linearized curvature

$$
R_{0 i 0 j}^{\operatorname{lin}}=-\frac{1}{2}\left(\partial_{i j} h_{00}+\partial_{00} h_{i j}-\partial_{0 i} h_{0 j}-\partial_{0 j} h_{0 i}\right) .
$$

The conservative, time-symmetric part of the tailtransported contribution to Eq. A11 is

$$
\begin{aligned}
& h_{i j}^{\mathrm{TT} \text { nonloc }}= \\
& +\frac{8 G^{2} M}{5 c^{8}} \int_{0}^{+\infty} \mathrm{d} \tau \ln \left(\frac{c|\tau|}{2 s}\right)\left[\mathcal{J}_{i j}^{(3)}(t+\tau)-\mathcal{J}_{i j}^{(3)}(t-\tau)\right] \\
& =-\frac{8 G^{2} M}{5 c^{8}} \operatorname{Pf}_{2 s / c} \int_{-\infty}^{+\infty} \frac{\mathrm{d} \tau}{|\tau|} \mathcal{J}_{i j}^{(2)}(t+\tau) \text {. }
\end{aligned}
$$

The elimination of the $h_{i j}^{\mathrm{TT}}$ field consists in replacing the complete solution (A9) of the TT field equation A8 in the Routhian (A7). At leading order, the effect of $h_{i j}^{\mathrm{TT} \text { nonloc }}$ is linear in $h_{i j}^{\mathrm{TT}}$ nonloc and should a priori come from the first and third terms in Eq. A77. Such a standard computation yields

$$
\begin{gathered}
{\left[R^{\text {nonloc }}\right]^{\text {standard }}=\int \mathrm{d}^{3} x\left[\frac{1}{4} \partial_{k} h_{i j}^{\mathrm{TT} \text { nonloc }} \partial_{k} h_{i j}^{\mathrm{TT} \text { loc PN }}\right.} \\
+\frac{1}{4} \partial_{k} h_{i j}^{\mathrm{TT} \text { loc PN }} \partial_{k} h_{i j}^{\mathrm{TT} \text { nonloc }} \\
\left.\quad-\frac{1}{4} h_{i j}^{\mathrm{TT} \text { nonloc }} J_{i j}\right], \quad(\mathrm{A} 14)
\end{gathered}
$$

where we exhibited the two separate (though trivially equal) contributions coming from the (spatial) kinetic term $\frac{1}{4}(\nabla h)^{2}$ in Eq. A7).

However, the explicit expression A13 of the nonlocal contribution to $h_{i j}^{\mathrm{TT}}$ shows, as already mentioned, that $h_{i j}^{\mathrm{TT}}$ nonloc is spatially independent, being a mere function of time. As a consequence the first two (equal) terms on the rhs of Eq. (A14) give simply a zero contribution so that this standard computation yields

$$
\left[R^{\text {nonloc }}\right]^{\text {standard }}=\int \mathrm{d}^{3} x\left[-\frac{1}{4} h_{i j}^{\mathrm{TT} \text { nonloc }} J_{i j}\right] .
$$

Actually, this answer is incorrect, because it does not agree with the one (securely based on the equations of motion) that we used in Ref. 16.

A formal way out of this contradiction was advocated, and used, in Ref. [21]. It consists in replacing the standard kinetic terms $(\partial h)^{2}$ in Eq. (A7) by $-h \square h$. If we apply this formal procedure to the spatial gradient terms in Eq. (A7) (which are the only relevant ones at this order $)$, i.e., $\frac{1}{4}\left(\partial_{k} h_{i j}^{\mathrm{TT}}\right)^{2} \rightarrow-\frac{1}{4} h_{i j}^{\mathrm{TT}} \Delta h_{i j}^{\mathrm{TT}}$, the result of replacing the complete solution (A9) in the Routhian now yields, instead of Eq. (A14), the new expression

$$
\begin{aligned}
& {\left[R^{\text {nonloc }}\right]^{\text {nonstandard }}=\int \mathrm{d}^{3} x\left[-\frac{1}{4} h_{i j}^{\mathrm{TT} \text { nonloc }} \Delta h_{i j}^{\mathrm{TT} \text { loc PN }}\right.} \\
& \left.-\frac{1}{4} h_{i j}^{\mathrm{TT} \text { loc PN }} \Delta h_{i j}^{\mathrm{TT} \text { nonloc }}-\frac{1}{4} h_{i j}^{\mathrm{TT} \text { nonloc }} J_{i j}\right] . \quad(\mathrm{A} 16)
\end{aligned}
$$

Formally, this is identical to Eq. A14 modulo a (spatial) integration by parts. However, if we use the fact that $h_{i j}^{\mathrm{TT}}$ nonloc is simply a function of time only, the second term on the rhs now yields a vanishing contribution, while the first one yields (upon using the field equation for $h_{i j}^{\mathrm{TT} \text { loc PN }}$ )

$$
\begin{aligned}
{\left[R^{\text {nonloc }}\right]^{\text {nonstandard }}=\int \mathrm{d}^{3} x } & {\left[\frac{1}{8} h_{i j}^{\mathrm{TT} \text { nonloc }} \delta_{i j}^{\mathrm{TT} k l} J_{k l}\right.} \\
& \left.-\frac{1}{4} h_{i j}^{\mathrm{TT} \text { nonloc }} J_{i j}\right] . \quad(\mathrm{A} 17)
\end{aligned}
$$

If we now formally integrate by parts the spatially nonlocal projections described by the TT projection kernel and decide that the spatial derivatives they contain yield zero on $h_{i j}^{\mathrm{TT} \text { nonloc }}(t)$, we further get

$$
\begin{gathered}
{\left[R^{\text {nonloc }}\right]_{\text {formal by parts }}^{\text {nonstandard }}=\int \mathrm{d}^{3} x\left[+\frac{1}{8} h_{i j}^{\mathrm{TT} \text { nonloc }} J_{i j}\right.} \\
\left.-\frac{1}{4} h_{i j}^{\mathrm{TT} \text { nonloc }} J_{i j}\right]=\int \mathrm{d}^{3} x\left[-\frac{1}{8} h_{i j}^{\mathrm{TT} \text { nonloc }} J_{i j}\right] .
\end{gathered}
$$

As we see, we now have half the value A15 given by using the standard kinetic terms. And this second result now agrees with the result given in our previous work. Indeed, the last result A18 only involves the spatial integral $\mathcal{J}_{i j}$, Eq. (A12), of $J_{i j}$. Inserting also the expression A13) of $h_{i j}^{\text {TT nonloc }}$ as a nonlocal integral of $\mathcal{J}_{i j}^{(2)}$ yields

$$
\begin{aligned}
& {\left[R^{\text {nonloc }}\right]^{\text {nonstandard }} }=+\frac{G^{2} M}{5 c^{8}} \mathcal{J}_{i j}(t) \\
& \times \operatorname{Pf}_{2 s / c} \int_{-\infty}^{+\infty} \frac{\mathrm{d} \tau}{|\tau|} \mathcal{J}_{i j}^{(2)}(t+\tau),
\end{aligned}
$$

so that the corresponding nonlocal contribution to the action reads (modulo an integration by parts with respect to time)

$$
\begin{aligned}
-\int R_{\text {nonstandard }}^{\text {nonloc }} \mathrm{d} t & =+\frac{G^{2} M}{5 c^{8}} \\
& \times \operatorname{Pf}_{2 s / c} \iint \frac{\mathrm{d} t \mathrm{~d} t^{\prime}}{\left|t^{\prime}-t\right|} \mathcal{J}_{i j}^{(1)}\left(t^{\prime}\right) \mathcal{J}_{i j}^{(1)}(t) .
\end{aligned}
$$


Here, we note that the integral $\mathcal{J}_{i j}$ of $J_{i j}$, Eq. A12), is the following function of positions and momenta:

$$
\mathcal{J}_{i j}=2 \sum_{a} \frac{p_{a\langle i} p_{a j\rangle}}{m_{a}}-2 G m_{1} m_{2} \frac{r_{12}^{\langle i} r_{12}^{j\rangle}}{r_{12}^{3}},
$$

which is simply the on-shell value of the second time derivative, say $\hat{I}_{i j}^{(2)}$ (in the notation used in Ref. [21]), of the quadrupole moment $I_{i j}=\sum_{a} m_{a} x_{a}^{\langle i} x_{a}^{j\rangle}$. We thereby see that, modulo the eventual order reduction of the ex- tra time derivative in $\mathcal{J}_{i j}^{(1)}=\frac{\mathrm{d}}{\mathrm{d} t} \hat{I}_{i j}^{(2)}$, the result A20 agrees with the nonlocal action of Ref. [16], as well as with the equivalent action of Ref. [21]. However, our explicit calculation above has given us two new results: 1) a reduced-action calculation similar to the one performed in harmonic coordinates in 21] can be done within the ADM formalism; and 2) such a calculation has, at best, a formal value because it is very sensitive to ill-controlled IR effects (spatial divergences) and can easily be conducted so as to give a wrong result, as exhibited in Eqs. (A14) and (A15), based on the standard $(\partial h)^{2}$ action.
[1] T. Damour, P. Jaranowski, and G. Schäfer, "Dimensional regularization of the gravitational interaction of point masses," Phys. Lett. B 513, 147 (2001) arXiv:gr-qc/0105038.

[2] Y. Itoh and T. Futamase, "New derivation of a third post-Newtonian equation of motion for relativistic compact binaries without ambiguity," Phys. Rev. D 68, 121501(R) (2003) arXiv:gr-qc/0310028.

[3] Y. Itoh, "Equation of motion for relativistic compact binaries with the strong field point particle limit: Third post-Newtonian order," Phys. Rev. D 69, 064018 (2004) arXiv:gr-qc/0310029.

[4] L. Blanchet, T. Damour, and G. Esposito-Farèse, "Dimensional regularization of the third post-Newtonian dynamics of point particles in harmonic coordinates," Phys. Rev. D 69, 124007 (2004) arXiv:gr-qc/0311052.

[5] S. Foffa and R. Sturani, "Effective field theory calculation of conservative binary dynamics at third post-Newtonian order," Phys. Rev. D 84, 044031 (2011) arXiv:1104.1122 [gr-qc]].

[6] T. Damour, "Gravitational self force in a Schwarzschild background and the effective one body formalism," Phys. Rev. D 81, 024017 (2010) arXiv:0910.5533 [gr-qc]].

[7] L. Blanchet, S. Detweiler, A. Le Tiec, and B. F. Whiting, "High-order post-Newtonian fit of the gravitational selfforce for circular orbits in the Schwarzschild geometry," Phys. Rev. D 81, 084033 (2010) arXiv:1002.0726 [gr-qc]].

[8] T. Damour (unpublished); cited in L. Barack, T. Damour, and N. Sago, "Precession effect of the gravitational self-force in a Schwarzschild spacetime and the effective one-body formalism," Phys. Rev. D 82, 084036 (2010) arXiv:1008.0935 [gr-qc]], which quoted and used some combinations of the $4 \mathrm{PN}$ and $5 \mathrm{PN}$ logarithmic contributions to $a(u)$ and $\bar{d}(u)$. The method used for deriving these results was published in Sec. IX A of Ref. [17].

[9] E. Barausse, A. Buonanno, and A. Le Tiec, "The complete non-spinning effective-one-body metric at linear order in the mass ratio," Phys. Rev. D 85, 064010 (2012) arXiv:1111.5610 [gr-qc]].

[10] P. Jaranowski and G. Schäfer, "Towards the fourth postNewtonian Hamiltonian for two-point-mass systems," Phys. Rev. D 86, 061503(R) (2012) arXiv:1207.5448 [grqc]].

[11] S. Foffa and R. Sturani, "Dynamics of the gravitational two-body problem at fourth post-Newtonian order and at quadratic order in the Newton constant," Phys. Rev. D 87, 064011 (2013) arXiv:1206.7087v2 [gr-qc]].
[12] P. Jaranowski and G. Schäfer, "Dimensional regularization of local singularities in the fourth postNewtonian two-point-mass Hamiltonian," Phys. Rev. D 87, 081503(R) (2013) arXiv:1303.3225 [gr-qc]].

[13] D. Bini and T. Damour, "Analytical determination of the two-body gravitational interaction potential at the fourth post-Newtonian approximation," Phys. Rev. D 87, 121501(R) (2013) arXiv:1305.4884 [gr-qc]].

[14] P. Jaranowski and G. Schäfer, "Derivation of local-intime fourth post-Newtonian ADM Hamiltonian for spinless compact binaries," Phys. Rev. D 92, 124043 (2015) arXiv:1508.01016 [gr-qc]].

[15] T. Ledvinka, G. Schäfer, and J. Bičák, "Relativistic closed-form Hamiltonian for many-body gravitating systems in the post-Minkowskian approximation," Phys. Rev. Lett. 100, 251101 (2008) arXiv:0807.0214 [gr-qc]]

[16] T. Damour, P. Jaranowski, and G. Schäfer, "Nonlocalin-time action for the fourth post-Newtonian conservative dynamics of two-body systems," Phys. Rev. D 89, 064058 (2014) arXiv:1401.4548 [gr-qc]].

[17] T. Damour, P. Jaranowski, and G. Schäfer, "Fourth postNewtonian effective one-body dynamics," Phys. Rev. D 91, 084024 (2015) arXiv:1502.07245 [gr-qc]].

[18] L. Blanchet and T. Damour, "Tail transported temporal correlations in the dynamics of a gravitating system," Phys. Rev. D 37, 1410 (1988).

[19] S. Foffa and R. Sturani, "Tail terms in gravitational radiation reaction via effective field theory," Phys. Rev. D 87, 044056 (2013) arXiv:1111.5488 [gr-qc]].

[20] C. R. Galley, A. K. Leibovich, R. A. Porto, and A. Ross, "The tail effect in gravitational radiationreaction: Time non-locality and renormalization group evolution," arXiv:1511.07379 [gr-qc].

[21] L. Bernard, L. Blanchet, A. Bohé, G. Faye, and S. Marsat, "Fokker action of non-spinning compact binaries at the fourth post-Newtonian approximation," arXiv:1512.02876v2 [gr-qc].

[22] A. Buonanno and T. Damour, "Effective one-body approach to general relativistic two-body dynamics," Phys. Rev. D 59, 084006 (1999) arXiv:gr-qc/9811091.

[23] A. Buonanno and T. Damour, "Transition from inspiral to plunge in binary black hole coalescences," Phys. Rev. D 62, 064015 (2000) arXiv:gr-qc/0001013.

[24] T. Damour, P. Jaranowski, and G. Schäfer, "On the determination of the last stable orbit for circular general relativistic binaries at the third post-Newtonian approximation," Phys. Rev. D 62, 084011 (2000) 
arXiv:gr-qc/0005034.

[25] T. Damour, "Coalescence of two spinning black holes: An effective one-body approach," Phys. Rev. D 64, 124013 (2001) arXiv:gr-qc/0103018.

[26] A. Le Tiec, L. Blanchet, and B. F. Whiting, "The first law of binary black hole mechanics in general relativity and post-Newtonian theory," Phys. Rev. D 85, 064039 (2012) arXiv:1111.5378 [gr-qc]].

[27] L. Blanchet, A. Buonanno, and A. Le Tiec, "First law of mechanics for black hole binaries with spins," Phys. Rev. D 87, 024030 (2013) arXiv:1211.1060 [gr-qc]].

[28] A. Le Tiec, "First law of mechanics for compact binaries on eccentric orbits," Phys. Rev. D 92, 084021 (2015) arXiv:1506.05648 [gr-qc]].

[29] S. Mano, H. Suzuki, and E. Takasugi, "Analytic solutions of the Regge-Wheeler equation and the postMinkowskian expansion," Prog. Theor. Phys. 96, 549 (1996) arXiv:gr-qc/9605057.

[30] S. Mano, H. Suzuki, and E. Takasugi, "Analytic solutions of the Teukolsky equation and their low frequency expansions," Prog. Theor. Phys. 95, 1079 (1996) arXiv:gr-qc/9603020.

[31] D. Bini, T. Damour, and A. Geralico, "Confirming and improving post-Newtonian and effective-one-body results from self-force computations along eccentric orbits around a Schwarzschild black hole," Phys. Rev. D 93, 064023 (2016) arXiv:1511.04533 [gr-qc]].

[32] S. Hopper, C. Kavanagh, and A. C. Ottewill, "Analytic self-force calculations in the post-Newtonian regime: eccentric orbits on a Schwarzschild background," Phys. Rev. D 93, 044010 (2016) arXiv:1512.01556 [gr-qc]].

[33] D. Bini, T. Damour, and A. Geralico, "New gravitational self-force analytical results for eccentric orbits around a Schwarzschild black hole," arXiv:1601.02988 [gr-qc].

[34] L. Barack, T. Damour, and N. Sago, "Precession effect of the gravitational self-force in a Schwarzschild spacetime and the effective one-body formalism," Phys. Rev. D 82, 084036 (2010) arXiv:1008.0935 [gr-qc]].

[35] M. van de Meent et al. (to be published).

[36] C. Kavanagh, A. C. Ottewill, and B. Wardell, "Analytical high-order post-Newtonian expansions for extreme mass ratio binaries," Phys. Rev. D 92, 084025 (2015) arXiv:1503.02334 [gr-qc]].

[37] N. K. Johnson-McDaniel, A. G. Shah and B. F. Whiting, "Experimental mathematics meets gravitational self-force," Phys. Rev. D 92, 044007 (2015) arXiv:1503.02638 [gr-qc]].

[38] A. G. Shah, J. L. Friedman and B. F. Whiting, "Finding high-order analytic post-Newtonian parameters from a high-precision numerical self-force calculation," Phys. Rev. D 89, 064042 (2014) arXiv:1312.1952 [gr-qc]].

[39] M. van de Meent and A. G. Shah, "Metric pertur- bations produced by eccentric equatorial orbits around a Kerr black hole," Phys. Rev. D 92, 064025 (2015) arXiv:1506.04755 [gr-qc]].

[40] S. Akcay and M. van de Meent, "Numerical computation of the EOB potential $q$ using self-force results," Phys. Rev. D 93, 064063 (2016) arXiv:1512.03392 [gr-qc]].

[41] G. Schäfer, "Acceleration-dependent lagrangians in general relativity," Phys. Lett. A 100, 128 (1984).

[42] T. Damour and G. Schäfer, "Lagrangians for $n$ point masses at the second post-Newtonian approximation of general relativity," Gen. Relativ. Gravit. 17, 879 (1985).

[43] J. Vines and É. É. Flanagan, "Is motion under the conservative self-force in black hole spacetimes an integrable Hamiltonian system?," Phys. Rev. D 92, 064039 (2015) arXiv:1503.04727 [gr-qc]].

[44] T. Damour, P. Jaranowski, and G. Schäfer, "Dynamical invariants for general relativistic two-body systems at the third post-Newtonian approximation," Phys. Rev. D 62, 044024 (2000) arXiv:gr-qc/9912092.

[45] T. Damour and G. Schäfer, "Redefinition of position variables and the reduction of higher order Lagrangians," J. Math. Phys. (N.Y.) 32, 127 (1991).

[46] D. Bini and T. Damour, "Detweiler's gauge-invariant redshift variable: Analytic determination of the nine and nine-and-a-half post-Newtonian self-force contributions," Phys. Rev. D 91, 064050 (2015) arXiv:1502.02450] [grqc]].

[47] P. Jaranowski and G. Schäfer, "Third post-Newtonian higher order ADM Hamilton dynamics for two-body point mass systems," Phys. Rev. D 57, 7274 (1998); 63, 029902(E) (2000) arXiv:gr-qc/9712075.

[48] L. Blanchet and G. Faye, "General relativistic dynamics of compact binaries at the third post-Newtonian order," Phys. Rev. D 63, 062005 (2001) arXiv:gr-qc/0007051.

[49] L. Blanchet, B. R. Iyer, and B. Joguet, "Gravitational waves from inspiralling compact binaries: Energy flux to third post-Newtonian order," Phys. Rev. D 65, 064005 (2002); 71, 129903(E) (2005) arXiv:gr-qc/0105098.

[50] A. D. Fokker, "Ein invarianter Variationssatz für die Bewegung mehrerer elektrischer Massenteilchen," Z. Phys. 58, 386 (1929).

[51] T. Damour and G. Esposito-Farèse, "Testing gravity to second post-Newtonian order: A field theory approach," Phys. Rev. D 53, 5541 (1996) arXiv:gr-qc/9506063.

[52] T. Damour, "Gravitational radiation reaction in the binary pulsar and the quadrupole formula controversy," Phys. Rev. Lett. 51, 1019 (1983).

[53] A. Pound, "Second-order perturbation theory: Problems on large scales," Phys. Rev. D 92, 104047 (2015) arXiv:1510.05172 [gr-qc]].

[54] G. Schäfer, "Reduced Hamiltonian formalism for generalrelativistic adiabatic fluids and applications," Astron. Nachr. 311, 213 (1990). 